TRANSACTIONS OF THE

AMERICAN MATHEMATICAL SOCIETY

Volume 358, Number 9, September 2006, Pages 4071-4097

S 0002-9947(05)03785-2

Article electronically published on October 31,2005

\title{
A MOMENT PROBLEM AND A FAMILY OF INTEGRAL EVALUATIONS
}

\author{
JACOB S. CHRISTIANSEN AND MOURAD E. H. ISMAIL
}

This paper is dedicated to Olav Njästad on the occasion of his seventieth birthday.

\begin{abstract}
We study the Al-Salam-Chihara polynomials when $q>1$. Several solutions of the associated moment problem are found, and the orthogonality relations lead to explicit evaluations of several integrals. The polynomials are shown to have raising and lowering operators and a second order operator equation of Sturm-Liouville type whose eigenvalues are found explicitly. We also derive new measures with respect to which the Ismail-Masson system of rational functions is biorthogonal. An integral representation of the right inverse of a divided difference operator is also obtained.
\end{abstract}

\section{INTRODUCTION}

In this work we shall follow the notation of Gasper and Rahman [10] or Andrews, Askey, and Roy $[3$ for basic hypergeometric series and use the theory of the moment problem as described in Akhiezer [1. Other useful references are [19] and 21]. A modern treatment is in the interesting article by Simon [20].

The best example of an indeterminate moment problem on the real line is the moment problem studied by Ismail and Masson in [13. The corresponding orthogonal polynomials, usually denoted $h_{n}(x \mid q)$, are called the $q^{-1}$-Hermite polynomials and satisfy the three-term recurrence relation

$$
2 x h_{n}(x \mid q)=h_{n+1}(x \mid q)+q^{-n}\left(1-q^{n}\right) h_{n-1}(x \mid q), \quad n \geq 0,
$$

with initial conditions $h_{-1}=0$ and $h_{0}=1$.

Askey 2 was the first to give an explicit weight function for the polynomials $\left\{h_{n}(x \mid q)\right\}$. Using the Askey-Roy $q$-beta integral [5],

$$
\begin{array}{r}
\int_{0}^{\infty} t^{c-1} \frac{(-a t,-b q / t ; q)_{\infty}}{(-t,-q / t ; q)_{\infty}} d t=\frac{\left(a b, q^{c}, q^{1-c} ; q\right)_{\infty}}{\left(q, a q^{-c}, b q^{c} ; q\right)_{\infty}} \frac{\pi}{\sin \pi c} \\
\left(c>0,|a|<q^{c},|b|<q^{-c}\right),
\end{array}
$$

Received by the editors March 30, 2003 and, in revised form, July 13, 2004.

2000 Mathematics Subject Classification. Primary 33D45, 44A60; Secondary 47B34.

Key words and phrases. Indeterminate moment problems, $q^{-1}$-Hermite polynomials, AlSalam-Chihara polynomials, biorthogonal rational functions, divided difference operators, raising and lowering operators, Bethe Ansatz equations, integral operators.

Part of this work was done while the first author was visiting the University of South Florida in Tampa, and he gratefully acknowledges the generous financial support from the private Danish foundation "Travelling Scholarship for Mathematicians" (Rejselegat for Matematikere) and the hospitality of the University of South Florida.

The second author's research was partially supported by NSF grant DMS 99-70865. 
he proved that

$$
\int_{-\infty}^{\infty} \frac{h_{m}(\sinh y \mid q) h_{n}(\sinh y \mid q)}{\left(-q e^{2 y},-q e^{-2 y} ; q\right)_{\infty}} d y=\log q^{-1}(q ; q)_{\infty}(q ; q)_{n} q^{-\left(\begin{array}{c}
n+1 \\
2
\end{array}\right)} \delta_{m, n} .
$$

Whenever $y$ occurs we shall always assume that

$$
x=\sinh y .
$$

In 1994 Ismail and Masson [13] considered the $q^{-1}$-Hermite polynomials in details. They established the generating function

$$
\sum_{n=0}^{\infty} \frac{q^{\left(\begin{array}{c}
n \\
2
\end{array}\right)}}{(q ; q)_{n}} h_{n}(x \mid q) t^{n}=\left(-t e^{y}, t e^{-y} ; q\right)_{\infty}, \quad t \in \mathbb{C},
$$

as well as the Poisson kernel

$$
\begin{aligned}
& \sum_{n=0}^{\infty} \frac{q^{\left(\begin{array}{c}
n \\
2
\end{array}\right)}}{(q ; q)_{n}} h_{n}(x \mid q) h_{n}\left(x^{\prime} \mid q\right) t^{n} \\
& \quad=\frac{\left(-t e^{y+y^{\prime}},-t e^{-y-y^{\prime}}, t e^{y-y^{\prime}}, t e^{-y+y^{\prime}} ; q\right)_{\infty}}{\left(t^{2} / q ; q\right)_{\infty}}, \quad|t|<\sqrt{q}
\end{aligned}
$$

Moreover they proved that

$$
\int_{-\infty}^{\infty} \prod_{j=1}^{4}\left(-t_{j} e^{y}, t_{j} e^{-y} ; q\right)_{\infty} d \psi(x)=\frac{\prod_{1 \leq j<k \leq 4}\left(-t_{j} t_{k} / q ; q\right)_{\infty}}{\left(t_{1} t_{2} t_{3} t_{4} / q^{3} ; q\right)_{\infty}}
$$

whenever $\psi$ is a solution to the moment problem. Since the integrand is the product of four generating functions for $\left\{h_{n}(x \mid q)\right\}$, the integral in (1.6) now plays the role played by the Askey-Wilson integral in the study of the continuous $q$-Hermite polynomials.

The Nevanlinna matrix was also computed in 13, and it is remarkable that all the $N$-extremal solutions were found explicitly. They have the form

$$
\nu_{a}=\frac{1}{\left(-a^{2},-q / a^{2}, q ; q\right)_{\infty}} \sum_{n=-\infty}^{\infty} a^{4 n}\left(1+a^{2} q^{2 n}\right) q^{n(2 n-1)} \varepsilon_{x_{n}(a)}, \quad q<a \leq 1,
$$

where

$$
x_{n}(a)=\frac{1}{2}\left(\frac{1}{a q^{n}}-a q^{n}\right)
$$

and $\varepsilon_{x}$ denotes the measure having only a unit mass at the point $x$. In addition, the absolutely continuous solutions with densities

$$
w(x ; a)=\frac{a}{\pi i} \frac{(-a \bar{a},-q / a \bar{a}, \bar{a} / a, q a / \bar{a}, q ; q)_{\infty}}{\left|\left(a e^{y},-a e^{-y},-q e^{y} / a, q e^{-y} / a ; q\right)_{\infty}\right|^{2}}, \quad x=\sinh y \in \mathbb{R},
$$

were derived along with more complicated solutions. To begin with, the parameter $a$ in (1.8) belongs to the set

$$
\left\{r e^{i \theta} \mid r>0,0<\theta<\pi / 2\right\} \cup\left\{r e^{i \theta} \mid 0<r \leq 1, \theta=\pi / 2\right\},
$$

but since $w(x ; a q)=w(x ; a)$, it suffices to consider

$$
a \in\left\{r e^{i \theta} \mid q<r \leq 1,0<\theta \leq \pi / 2\right\} .
$$

We stress that no value of $a$ gives the Askey weight function appearing in (1.3). 
In the Nevanlinna parametrization, which gives a one-to-one correspondence between the set of Pick functions (including $\infty$ ) and the set of solutions to an indeterminate moment problem on the real line, the $N$-extremal solutions correspond to the Pick function being a real constant (or $\infty$ ). When the Pick function is a complex constant (in the open upper half-plane), the corresponding solutions are known to be absolutely continuous; see [6] and [13. The solutions in (1.8) are exactly of that kind.

The continuous $q$-Hermite polynomials belong to the Askey-scheme as a special case of the Askey-Wilson polynomials when all four parameters are zero; see [17. Halfway between Askey-Wilson and continuous $q$-Hermite we find the Al-SalamChihara polynomials with two free parameters. These polynomials are studied in 4 and when $q>1$, the associated moment problem is determinate or indeterminate depending on the parameters. In the indeterminate case the Nevanlinna matrix was computed in 9 ] but no explicit solutions were derived. In this paper we restrict ourselves to the symmetric case of the Al-Salam-Chihara polynomials when $q>1$. The analysis in the symmetric case simplifies a great deal, and a simple weight function for the polynomials can be found directly from the Nevanlinna matrix.

The paper is organized as follows. In Section 2 we present the Al-Salam-Chihara polynomials and consider the symmetric case when $q>1$. For convenience, we set $p=1 / q$ and besides $p$ there is only one parameter left. This parameter will be called $\beta$, and we point out how two special values, namely $\beta=0$ and $\beta=1 / p$, lead to the polynomials $\left\{h_{n}(x \mid q)\right\}$. In Section 3 we give an explicit expression for the Nevanlinna matrix based on the results in [9. On one hand the Nevanlinna matrix remains too complicated to give us the $N$-extremal solutions, as will be explained in Section 4. On the other hand, the Nevanlinna matrix is simple enough to lead to an explicit weight function, the function $v(x ; \beta)$ in $(5.1)$, and the corresponding orthogonality relation is given in Section [5. In the same section we derive a new family of absolutely continuous solutions to the $q^{-1}$-Hermite moment problem.

When $x \in \mathbb{R}$, we use the parameterization $x=\sinh y$. When $f$ is a function defined on $\mathbb{R}$, one can think of $f(x)$ as a function of $e^{y}$. We denote by $\breve{f}$ the function

$$
\breve{f}\left(e^{y}\right)=f(x)
$$

and the divided difference operator $\mathcal{D}_{q}$ given by

$$
\left(\mathcal{D}_{q} f\right)(x)=\frac{\breve{f}\left(q^{1 / 2} e^{y}\right)-\breve{f}\left(q^{-1 / 2} e^{y}\right)}{\left(q^{1 / 2}-q^{-1 / 2}\right) \cosh y}
$$

was introduced by Ismail in [11. Note that if we set $e(x)=x$, then the denominator can also be written as

$$
\breve{e}\left(q^{1 / 2} e^{y}\right)-\breve{e}\left(q^{-1 / 2} e^{y}\right) .
$$

It was proved in [11] that $\mathcal{D}_{q}$ is a lowering operator for the $q^{-1}$-Hermite polynomials.

We find a family of weight functions that lead to the same raising and lowering operators for the polynomials $\left\{h_{n}(x \mid q)\right\}$. Combining the lowering and raising operators one can obtain a $q$-Sturm-Liouville equation from which the orthogonality follows using Ismail's $q$-analogue of integration by parts [11. Besides the Askey weight function (in (1.3) ), the family also contains the special case $\beta=1 / p$ of the weight function $v(x ; \beta)$. 
In Section 6 we construct a family of discrete solutions from the weight function $v(x ; \beta)$. These solutions are not $N$-extremal, though they are supported on the same $p$-quadratic grid as the $N$-extremal solutions to the $q^{-1}$-Hermite moment problem. A possible way to verify this is through the Poisson kernel which we shall derive from a bilinear generating function established in Section 7 .

One way of reaching the Al-Salam-Chihara polynomials when $q>1$ is to start out with the $q^{-1}$-Hermite polynomials and use a simple procedure of attaching generating functions to measures. This procedure is explained in [7, and the next step takes us to the biorthogonal rational functions with four parameters studied by Ismail and Masson in 13 . In Section 8 we show how solutions to the moment problem lead to biorthogonality relations for the rational functions when certain restrictions on the last two parameters are fulfilled.

In Section 9 we obtain a $p$-Sturm-Liouville equation from lowering and raising operators. The weight function $v(x ; \beta)$ appears in the raising operator, but the $p$-Sturm-Liouville equation can be written in a form independent of $v(x ; \beta)$. We use this form to derive a system of $n$ nonlinear equations satisfied by the zeros of the polynomials. This is a typical example of Bethe Ansatz equations; see the Bethe Ansatz for the XXZ model in 18 and 12. In Section 10 we consider the divided difference operator $\mathcal{D}_{q}$ as a bounded operator on the $L^{2}$-spaces of the $N$ extremal solutions. The right inverse $\mathcal{D}_{q}^{-1}$ is identified as an integral operator, and we find the kernel explicitly. This is the $q>1$ version of a result in 8 . The kernel for the inverse of the Askey-Wilson operator over the $L^{2}$-space weighted by the Askey-Wilson weight function is in 14.

\section{The Al-Salam-Chinara polynomials}

The Al-Salam-Chihara polynomials $Q_{n}(x):=Q_{n}(x ; a, b \mid q)$ are generated by the three-term recurrence relation

$$
2 x Q_{n}(x)=Q_{n+1}(x)+(a+b) q^{n} Q_{n}(x)+\left(1-q^{n}\right)\left(1-a b q^{n-1}\right) Q_{n-1}(x), \quad n \geq 0,
$$

with initial conditions $Q_{-1}=0$ and $Q_{0}=1$; see for example [17. They are orthogonal with respect to a positive measure (with bounded support) on $\mathbb{R}$ if $a+b \in \mathbb{R}, a b<1$ and $0<q<1$. In the case $q>1$, the polynomials are orthogonal on the imaginary axis (for suitable values of $a$ and $b$ ), so we replace $x$ by $i x$ in order to obtain orthogonality on the real line. Indeed, with $p=1 / q$, the polynomials

$$
\widetilde{Q}_{n}(x)=\frac{i^{n} p^{\left(\begin{array}{c}
n \\
2
\end{array}\right)}}{(p ; p)_{n}} Q_{n}(i x / 2 ; a, b \mid p)
$$

satisfy the three-term recurrence relation

$$
\left(1-p^{n+1}\right) \widetilde{Q}_{n+1}(x)=\left(-i(a+b)-x p^{n}\right) \widetilde{Q}_{n}(x)-\left(-a b+p^{n-1}\right) \widetilde{Q}_{n-1}(x)
$$

and are therefore orthogonal with respect to a positive measure on $\mathbb{R}$ when $a+b \in$ $i \mathbb{R}, a b \leq 0$ and $0<p<1$. The polynomials in (2.1) are a special case of the polynomials $v_{n}(x)$ studied in [4] and [9. The parametrization, however, is slightly different, and we have to identify $a$ and $b$ from [4] and [9] with $-i(a+b)$ and $-a b$, respectively. The parameter $c$ is set to be -1 here.

In this paper we will study the special situation where $a=-b=\sqrt{\beta}$ for some $\beta \geq 0$. The motivation is simply to obtain symmetry. It is convenient to replace $x$ 
by $-2 x$, so our starting point is the polynomials $Q_{n}(x ; \beta)$ generated by the threeterm recurrence relation

$$
2 x p^{n} Q_{n}(x ; \beta)=\left(1-p^{n+1}\right) Q_{n+1}(x ; \beta)+\left(\beta+p^{n-1}\right) Q_{n-1}(x ; \beta), \quad n \geq 0,
$$

with initial conditions $Q_{-1}=0$ and $Q_{0}=1$. In accordance with [7] (set $t_{1}=-t_{2}=$ $i q \sqrt{\beta}$ and replace $q$ by $p$ ), the polynomials $Q_{n}(x ; \beta)$ are explicitly given by

$$
\begin{aligned}
Q_{n}(x ; \beta) & =\frac{\left(-i e^{-y} / \sqrt{\beta} ; p\right)_{n}}{(p ; p)_{n}}(i \sqrt{\beta})^{n}{ }_{2} \phi_{1}\left(\begin{array}{c}
p^{-n},-i e^{y} / \sqrt{\beta} \\
i \sqrt{\beta} e^{y} / p^{n-1}
\end{array} \mid p,-i p \sqrt{\beta} e^{y}\right) \\
& =\frac{(-1 / \beta ; p)_{n}}{(p ; p)_{n}}(i \sqrt{\beta})^{n} \sum_{k=0}^{n}(-1)^{k}\left[\begin{array}{c}
n \\
k
\end{array}\right]_{p} \frac{\left(i e^{-y} / \sqrt{\beta},-i e^{y} / \sqrt{\beta} ; p\right)_{k}}{(-1 / \beta ; p)_{k}},
\end{aligned}
$$

where $x=\sinh y$. Since the polynomials

$$
h_{n}(x ; \beta)=\frac{(p ; p)_{n}}{p^{\left(\begin{array}{c}
n \\
2
\end{array}\right)}} Q_{n}(x ; \beta)
$$

satisfy the three-term recurrence relation

$$
2 x h_{n}(x ; \beta)=h_{n+1}(x ; \beta)+\left(p^{-2 n+1} \beta+p^{-n}\right)\left(1-p^{n}\right) h_{n-1}(x ; \beta),
$$

we immediately see that the special case $\beta=0$ of our polynomials is $\left\{h_{n}(x \mid p)\right\}$. Furthermore, we observe that the special case $\beta=1 / p$ corresponds to the polynomials $\left\{h_{n}\left(x \mid p^{2}\right)\right\}$. Throughout the paper we shall always try to have these special cases in mind. Certainly, this will throw more light on the $q^{-1}$-Hermite polynomials as well.

\section{The Nevanlinna matrix}

According to Theorem 3.2 in 4, the moment problem associated with the polynomials $Q_{n}(x ; \beta)$ is indeterminate for $\beta \geq 0$. The entire functions from the Nevanlinna matrix were computed in [9]. The first step in the computation was to establish the generating functions

$$
\sum_{n=0}^{\infty} Q_{n}(x ; \beta) t^{n}=\frac{\left(t e^{-y},-t e^{y} ; p\right)_{\infty}}{\left(-t^{2} \beta ; p^{2}\right)_{\infty}}, \quad|t|<1 / \sqrt{\beta}
$$

and

$$
\begin{aligned}
& \sum_{n=0}^{\infty} Q_{n}^{*}(x ; \beta) t^{n}=\frac{2 p t}{1+t^{2} \beta}{ }_{3} \phi_{2}\left(\begin{array}{c}
t e^{-y},-t e^{y}, p \\
i p t \sqrt{\beta},-i p t \sqrt{\beta}
\end{array} \mid p, p\right) \\
& =2 t \sum_{n=0}^{\infty} \frac{\left(t e^{-y},-t e^{y} ; p\right)_{n}}{\left(-t^{2} \beta ; p^{2}\right)_{n+1}} p^{n+1}, \quad|t|<1 / \sqrt{\beta},
\end{aligned}
$$

where $Q_{n}^{*}(x ; \beta)$ denotes the numerator polynomials, that is, the polynomials generated by the three-term recurrence relation (2.2) with initial conditions $Q_{0}^{*}=0$ and $Q_{1}^{*}=2 p /(1-p)$. Darboux's method was then applied to find the asymptotic behavior of $Q_{n}$ and $Q_{n}^{*}$ as $n \rightarrow \infty$. In our case, the expressions for the functions 
$A, B, C$, and $D$ reduce to

$$
\begin{aligned}
& A(x ; \beta)=-\frac{2}{\sqrt{\beta}} \frac{(p ; p)_{\infty}}{(-1 / \beta ; p)_{\infty}} S(0) S(x) \sin (\eta(x)-\eta(0)), \\
& B(x ; \beta)=-\frac{(p ; p)_{\infty}}{(-1 / \beta ; p)_{\infty}} S(0) R(x) \cos (\zeta(x)-\eta(0)), \\
& C(x ; \beta)=\frac{(p ; p)_{\infty}}{(-1 / \beta ; p)_{\infty}} R(0) S(x) \cos (\eta(x)-\zeta(0)), \\
& D(x ; \beta)=-\frac{\sqrt{\beta}}{2} \frac{(p ; p)_{\infty}}{(-1 / \beta ; p)_{\infty}} R(0) R(x) \sin (\zeta(x)-\zeta(0)),
\end{aligned}
$$

where

$$
R(x) e^{i \zeta(x)}=\frac{\left(i e^{-y} / \sqrt{\beta},-i e^{y} / \sqrt{\beta} ; p\right)_{\infty}}{\left(p^{2} ; p^{2}\right)_{\infty}}
$$

and

$$
\begin{aligned}
S(x) e^{i \eta(x)} & ={ }_{2} \phi_{1}\left(\begin{array}{c}
i e^{-y} / \sqrt{\beta},-i e^{y} / \sqrt{\beta} \\
-p
\end{array} \mid p, p\right) \\
& =\sum_{n=0}^{\infty} \frac{\left(i e^{-y} / \sqrt{\beta},-i e^{y} / \sqrt{\beta} ; p\right)_{n}}{\left(p^{2} ; p^{2}\right)_{n}} p^{n}
\end{aligned}
$$

for $x \in \mathbb{R}$. It is assumed that $\zeta(x), \eta(x) \in \mathbb{R}$ and $R(x), S(x)>0$. In particular, we have

$$
R(0)=\frac{\left(-1 / \beta ; p^{2}\right)_{\infty}}{\left(p^{2} ; p^{2}\right)_{\infty}}, \quad S(0)=\sum_{n=0}^{\infty} \frac{\left(-1 / \beta ; p^{2}\right)_{n}}{\left(p^{2} ; p^{2}\right)_{n}} p^{n}=\frac{\left(-p / \beta ; p^{2}\right)_{\infty}}{\left(p ; p^{2}\right)_{\infty}}
$$

and

$$
\zeta(0)=\eta(0)=0 .
$$

So the expressions reduce further to the more convenient forms

$$
\begin{aligned}
& A(x ; \beta)=-\frac{2}{\sqrt{\beta}} \frac{\left(p^{2} ; p^{2}\right)_{\infty}}{\left(-1 / \beta ; p^{2}\right)_{\infty}} S(x) \sin (\eta(x)), \\
& B(x ; \beta)=-\frac{\left(p^{2} ; p^{2}\right)_{\infty}}{\left(-1 / \beta ; p^{2}\right)_{\infty}} R(x) \cos (\zeta(x)), \\
& C(x ; \beta)=\frac{\left(p ; p^{2}\right)_{\infty}}{\left(-p / \beta ; p^{2}\right)_{\infty}} S(x) \cos (\eta(x)), \\
& D(x ; \beta)=-\frac{\sqrt{\beta}}{2} \frac{\left(p ; p^{2}\right)_{\infty}}{\left(-p / \beta ; p^{2}\right)_{\infty}} R(x) \sin (\zeta(x)),
\end{aligned}
$$

for $x \in \mathbb{R}$. Hence, the Stieltjes transform of the solution $\mu_{\varphi}$ corresponding to the Pick function $\varphi$ in the Nevanlinna parametrization is given by

$$
\begin{aligned}
& \int_{\mathbb{R}} \frac{1}{t-x} d \mu_{\varphi}(t) \\
& =\frac{S(x)}{R(x)} \frac{4\left(-p / \beta, p^{2} ; p^{2}\right)_{\infty} \sin (\eta(x)) \varphi(x)+2 \sqrt{\beta}\left(-1 / \beta, p ; p^{2}\right)_{\infty} \cos (\eta(x))}{\beta\left(-1 / \beta, p ; p^{2}\right)_{\infty} \sin (\zeta(x))-2 \sqrt{\beta}\left(-p / \beta, p^{2} ; p^{2}\right)_{\infty} \cos (\zeta(x)) \varphi(x)} .
\end{aligned}
$$




\section{4. $N$-eXtremal SOLutions}

In the search for the $N$-extremal solutions $\mu_{t}^{(\beta)}, t \in \mathbb{R} \cup\{\infty\}$, it is convenient to write the parameter $t$ as

$$
t=\frac{D(u ; \beta)}{B(u ; \beta)}
$$

for $u$ belonging to, say, the interval $\left(-x_{1}, x_{1}\right]$, where $x_{1}$ is the smallest positive zero of $B$. With this parametrization, the Stieltjes transform in (3.3) takes the form

$$
\begin{aligned}
\int_{\mathbb{R}} \frac{1}{t-x} d \nu_{u}^{(\beta)}(t) & =\frac{2}{\sqrt{\beta}} \frac{S(x)}{R(x)} \frac{\sin (\eta(x)) \sin (\zeta(u))+\cos (\eta(x)) \cos (\zeta(u))}{\sin (\zeta(x)) \cos (\zeta(u))+\cos (\zeta(x)) \sin (\zeta(u))} \\
& =\frac{2}{\sqrt{\beta}} \frac{S(x)}{R(x)} \frac{\cos (\eta(x)-\zeta(u))}{\sin (\zeta(x)+\zeta(u))}
\end{aligned}
$$

so the $N$-extremal solution $\nu_{u}^{(\beta)}$ is supported on the set of real $x$ 's for which

$$
\zeta(x)-\zeta(u) \in \pi \mathbb{Z} .
$$

In other words, if we set $u=\sinh v$, then $x=\sinh y$ belongs to the support of $\nu_{u}^{(\beta)}$ if and only if

$$
\left(i e^{-y} / \sqrt{\beta},-i e^{-v} / \sqrt{\beta},-i e^{y} / \sqrt{\beta}, i e^{v} / \sqrt{\beta} ; p\right)_{\infty} \in \mathbb{R} .
$$

However, it seems impossible to solve the above equations explicitly except for the trivial solution $x=u$. In the special case $u=0$, for instance, we have to know exactly when

$$
\left(i e^{-y} / \sqrt{\beta},-i e^{y} / \sqrt{\beta} ; p\right)_{\infty} \in \mathbb{R}
$$

and even for $\beta=1$, this comes to find the values of $t \in i \mathbb{R}_{+}$for which

$$
\operatorname{Im}\left((t, 1 / t ; p)_{\infty}\right)=0 .
$$

\section{Absolutely continuous solutions}

For one particular Pick function we are able to find the corresponding solution explicitly. Observe that

$$
R^{2}(x)=\frac{\left(-e^{2 y} / \beta,-e^{-2 y} / \beta ; p^{2}\right)_{\infty}}{\left(p^{2} ; p^{2}\right)_{\infty}^{2}}
$$

so that $B^{2}$ and $D^{2}$ can be written as

$$
B^{2}(x ; \beta)=\frac{1}{\left(-1 / \beta ; p^{2}\right)_{\infty}^{2}}\left(-e^{2 y} / \beta,-e^{-2 y} / \beta ; p^{2}\right)_{\infty} \cos ^{2}(\zeta(x))
$$

and

$$
D^{2}(x ; \beta)=\frac{\beta}{4} \frac{\left(p ; p^{2}\right)_{\infty}^{2}}{\left(-p / \beta, p^{2} ; p^{2}\right)_{\infty}^{2}}\left(-e^{2 y} / \beta,-e^{-2 y} / \beta ; p^{2}\right)_{\infty} \sin ^{2}(\zeta(x)) .
$$

For the particular choice

$$
\gamma=\frac{\sqrt{\beta}}{2} \frac{\left(-1 / \beta, p ; p^{2}\right)_{\infty}}{\left(-p / \beta, p^{2} ; p^{2}\right)_{\infty}}
$$

the absolutely continuous solution $\mu_{i \gamma}$ with density

$$
\frac{\gamma / \pi}{D^{2}(x ; \beta)+\gamma^{2} B^{2}(x ; \beta)}, \quad x \in \mathbb{R},
$$


has the form $d \mu_{i \gamma}=v(x ; \beta) d x$, where

$$
v(x ; \beta)=\frac{\left(p^{2} ; p^{2}\right)_{\infty}}{\left(p ; p^{2}\right)_{\infty}} \frac{(-1 / \beta ; p)_{\infty}}{\pi \sqrt{\beta}} \frac{2}{\left(-e^{2 y} / \beta,-e^{-2 y} / \beta ; p^{2}\right)_{\infty}},
$$

$x=\sinh y \in \mathbb{R}$. We state this result as a theorem.

Theorem 5.1. The polynomials $Q_{n}(x ; \beta)$ are orthogonal with respect to the weight function

$$
\frac{1}{\left(-e^{2 y} / \beta,-e^{-2 y} / \beta ; p^{2}\right)_{\infty}}, \quad x=\sinh y \in \mathbb{R},
$$

and the orthogonality relation is

$$
\begin{aligned}
2 \int_{\mathbb{R}} & \frac{Q_{n}(\sinh y ; \beta) Q_{m}(\sinh y ; \beta)}{\left(-e^{2 y} / \beta,-e^{-2 y} / \beta ; p^{2}\right)_{\infty}} \cosh y d y \\
& =\frac{\pi \sqrt{\beta}}{(-1 / \beta ; p)_{\infty}} \frac{\left(p ; p^{2}\right)_{\infty}}{\left(p^{2} ; p^{2}\right)_{\infty}} \frac{\beta^{n}(-1 / \beta ; p)_{n}}{p^{n}(p ; p)_{n}} \delta_{n, m} .
\end{aligned}
$$

Proof. We only have to check that the orthogonality relation is correct. It follows from the three-term recurrence relation (2.2) that the polynomials

$$
P_{n}(x ; \beta)=\sqrt{\frac{p^{n}(p ; p)_{n}}{\beta^{n}(-1 / \beta ; p)_{n}}} Q_{n}(x ; \beta)
$$

are orthonormal. As a matter of fact, they satisfy the three-term recurrence relation

$$
x P_{n}(x ; \beta)=\frac{\sqrt{\left(1-p^{n+1}\right)\left(\beta+p^{n}\right)}}{2 p^{n+1 / 2}} P_{n+1}(x ; \beta)+\frac{\sqrt{\left(1-p^{n}\right)\left(\beta+p^{n-1}\right)}}{2 p^{n-1 / 2}} P_{n-1}(x ; \beta)
$$

with initial conditions

$$
P_{0}(x ; \beta)=1 \quad \text { and } \quad P_{1}(x ; \beta)=\frac{2 x \sqrt{p}}{\sqrt{(1-p)(1+\beta)}} .
$$

Therefore, we have the orthogonality relation

$$
\int_{\mathbb{R}} P_{n}(x ; \beta) P_{m}(x ; \beta) v(x ; \beta) d x=\delta_{n m}
$$

or, equivalently,

$$
\int_{\mathbb{R}} Q_{n}(x ; \beta) Q_{m}(x ; \beta) v(x ; \beta) d x=\frac{\beta^{n}(-1 / \beta ; p)_{n}}{p^{n}(p ; p)_{n}} \delta_{n m},
$$

and the result follows immediately.

The special case $\beta=1 / p$ of (5.1) leads directly to an absolutely continuous solution to the $q^{-1}$-Hermite moment problem. Replace $p^{2}$ by $q$ to obtain the density

$$
w(x)=q^{1 / 4} \frac{1}{\pi} \frac{(q ; q)_{\infty}}{(\sqrt{q} ; q)_{\infty}^{2}} \frac{2}{\left(-\sqrt{q} e^{2 y},-\sqrt{q} e^{-2 y} ; q\right)_{\infty}}, \quad x=\sinh y .
$$

Moreover, if we set $\beta=c p^{2 n}$ for fixed $c \in(0,1]$ and let $n \rightarrow \infty$, we obtain the densities

$$
w_{c}(x)=\frac{1}{\pi \sqrt{c}} \frac{\left(q^{2} ; q^{2}\right)_{\infty}}{\left(q ; q^{2}\right)_{\infty}} \frac{2(-1 / c,-c q ; q)_{\infty}}{\left(-e^{2 y} / c,-c e^{2 y} q^{2},-e^{-2 y} / c,-c e^{-2 y} q^{2} ; q^{2}\right)_{\infty}}
$$


after replacing $p$ by $q$. To see this, note that

$$
\begin{aligned}
& \frac{1}{\sqrt{c p^{2 n}}} \frac{\left(-1 / c p^{2 n} ; p\right)_{\infty}}{\left(-e^{2 y} / c p^{2 n},-e^{-2 y} / c p^{2 n} ; p^{2}\right)_{\infty}} \\
& =\frac{1}{\sqrt{c}} \frac{(-1 / c ; p)_{\infty}}{\left(-e^{2 y} / c,-e^{-2 y} / c ; p^{2}\right)_{\infty}} \frac{\left(-1 / c p^{2 n} ; p\right)_{2 n}}{p^{n}\left(-e^{2 y} / c p^{2 n},-e^{-2 y} / c p^{2 n} ; p^{2}\right)_{n}} \\
& =\frac{1}{\sqrt{c}} \frac{(-1 / c ; p)_{\infty}}{\left(-e^{2 y} / c,-e^{-2 y} / c ; p^{2}\right)_{\infty}} \frac{(-c p ; p)_{2 n}}{\left(-c e^{2 y} p^{2},-c e^{-2 y} p^{2} ; p^{2}\right)_{n}} \\
& \rightarrow \frac{1}{\sqrt{c}} \frac{(-1 / c,-c p ; p)_{\infty}}{\left(-e^{2 y} / c,-e^{-2 y} / c,-c e^{2 y} p^{2},-c e^{-2 y} p^{2} ; p^{2}\right)_{\infty}}
\end{aligned}
$$

as $n \rightarrow \infty$. By construction, we have $w_{c q^{2}}(x)=w_{c}(x)\left(\right.$ or $\left.w_{c q}(x)=w_{c / q}(x)\right)$ and since

$$
a(-1 / a,-a q ; q)_{\infty}=(-a,-q / a ; q)_{\infty} \text { for } a \neq 0,
$$

we also have $w_{1 / c}(x)=w_{c}(x)$. Therefore, it suffices to consider the case $q \leq c \leq 1$. Note that $w_{c}(x)$ reduces to $w(x)$ when $c=\sqrt{q}$ (or $1 / \sqrt{q}$ ).

The probability densities $w_{c}(x)$ are not new solutions. They are special cases of the densities $w(x ; a)$ in (1.8). Set $a=i q^{1 / 4}$ to obtain the density $w(x)$ and note that the densities $w_{c}(x)$ exactly correspond to $w(x ; a)$ when $a=i \gamma$ with $q<\gamma \leq 1$.

The orthogonality relation in (1.3) contains the probability density

$$
\tilde{w}(x)=\frac{1}{\log q^{-1}(q ; q)_{\infty}} \frac{2 e^{y}}{\left(-e^{2 y},-q e^{-2 y} ; q\right)_{\infty}}, \quad x=\sinh y,
$$

and the similarity to $w(x)$ in $(5.3)$ is striking. It turns out that

$$
h_{n+1}(x \mid q)=-\frac{1-q}{2 q^{1+n / 2} f(x)} \mathcal{D}_{q}\left(f(x) h_{n}(x \mid q)\right), \quad n \geq 0,
$$

for $f=w$ as well as $f=\tilde{w}$; cf. Theorem 5.2 below. So both $w(x)$ and $\tilde{w}(x)$ give rise to a raising operator for the $q^{-1}$-Hermite polynomials. With respect to the inner product

$$
\langle f, g\rangle=\int_{\mathbb{R}} f(x) \overline{g(x)} \frac{d x}{\sqrt{1+x^{2}}}
$$

on $L^{2}\left(\mathbb{R}, 1 / \sqrt{1+x^{2}}\right)$, the following rule for integration by parts applies

$$
\left\langle\mathcal{D}_{q} f, g\right\rangle=-\left\langle f, \sqrt{1+x^{2}} \mathcal{D}_{q}\left(g(x) / \sqrt{1+x^{2}}\right)\right\rangle ;
$$

see [11] for details. Combining the raising operator in (5.6) with the lowering operator

$$
\mathcal{D}_{q} h_{n}(x \mid q)=2 q^{(1-n) / 2} \frac{1-q^{n}}{1-q} h_{n-1}(x \mid q), \quad n \geq 0,
$$

which can be obtained from the generating function in (1.4), we are led to the $q$-Sturm-Liouville equation

$$
\mathcal{D}_{q}\left(f(x) \mathcal{D}_{q} h_{n}(x \mid q)\right)+4 q \frac{1-q^{n}}{(1-q)^{2}} f(x) h_{n}(x \mid q)=0, \quad n \geq 0 .
$$

Again, $f=w$ or $f=\tilde{w}$. The eigenvalues $4 q\left(1-q^{n}\right) /(1-q)^{2}$ are distinct and positive. This indicates that the operator $T$ defined by

$$
T \phi(x)=-\frac{1}{f(x)} \mathcal{D}_{q}\left(f(x) \mathcal{D}_{q} \phi(x)\right)
$$


is positive on the weighted Hilbert space $L^{2}(\mathbb{R}, f(x))$. Indeed this follows from (5.7).

The fact that the $q^{-1}$-Hermite polynomials are orthogonal with respect to $\tilde{w}(x)$ and $w(x)$ can now be obtained from (5.9) just by using integration by parts as described in (5.7). For more details, the reader is referred to the proof of Theorem 2.4 in [11.

We shall now describe a more general set up.

Theorem 5.2. Let $f_{c}$ denote the function given by

$$
f_{c}(x)=\frac{e^{y(2 c-1)}}{\left(-q^{1-c} e^{2 y},-q^{c} e^{-2 y} ; q\right)_{\infty}}, \quad x=\sinh y \in \mathbb{R} .
$$

For each $c \in \mathbb{R}$, we have the following raising operator for the $q^{-1}$-Hermite polynomials

$$
h_{n+1}(x \mid q)=-\frac{1-q}{2 q^{1+n / 2} f_{c}(x)} \mathcal{D}_{q}\left(f_{c}(x) h_{n}(x \mid q)\right), \quad n \geq 0 .
$$

Proof. We have to prove that (5.6) remains valid when $f$ is replaced by $f_{c}$. This is more or less a repetition of the proof of Theorem 2.1 in [11]. From the generating function in (1.4) a straightforward computation gives

$$
\begin{aligned}
\frac{1}{f_{c}(x)} & \sum_{n=0}^{\infty} \frac{q^{\left(\begin{array}{c}
n \\
2
\end{array}\right)}}{(q ; q)_{n}} t^{n} \mathcal{D}_{q}\left(f_{c}(x) h_{n}(x \mid q)\right) \\
& =\frac{2 q^{3 / 2}}{t(1-q)}\left\{\left(-t e^{y} \sqrt{q}, t e^{-y} \sqrt{q} ; q\right)_{\infty}-\left(-t e^{y} / \sqrt{q}, t e^{-y} / \sqrt{q} ; q\right)_{\infty}\right\}
\end{aligned}
$$

or

$$
\begin{aligned}
\frac{1}{f_{c}(x)} & \sum_{n=0}^{\infty} \frac{q^{\left(\begin{array}{c}
n \\
2
\end{array}\right)}}{(q ; q)_{n}} t^{n+1} \mathcal{D}_{q}\left(f_{c}(x) h_{n}(x \mid q)\right) \\
& =\frac{2 q^{3 / 2}}{1-q} \sum_{n=0}^{\infty} \frac{q^{\left(\begin{array}{c}
n \\
2
\end{array}\right)}}{(q ; q)_{n}} h_{n}(x \mid q) t^{n}\left(q^{n / 2}-q^{-n / 2}\right) \\
& =-\frac{2 q^{3 / 2}}{1-q} \sum_{n=1}^{\infty} \frac{q^{n^{2} / 2-n}}{(q ; q)_{n-1}} h_{n}(x \mid q) t^{n} \\
& =-\frac{2 q}{1-q} \sum_{n=0}^{\infty} \frac{q^{n^{2} / 2}}{(q ; q)_{n}} h_{n+1}(x \mid q) t^{n+1} .
\end{aligned}
$$

Equating the coefficients of $t^{n+1}$ now leads to the desired raising operator.

Corollary 5.3. The absolutely continuous measure with density

$$
v_{c}(x)=q^{c(1-c)} \frac{\sin \pi c}{\pi} \frac{(q ; q)_{\infty}}{\left(q^{c}, q^{1-c} ; q\right)_{\infty}} \frac{2 e^{y(2 c-1)}}{\left(-q^{1-c} e^{2 y},-q^{c} e^{-2 y} ; q\right)_{\infty}}, \quad x=\sinh y,
$$

is solution to the $q^{-1}$-Hermite moment problem.

Proof. Since $v_{c}$ satisfies the $q$-Sturm-Liouville equation (5.9), it is only left to verify that

$$
\int_{\mathbb{R}} v_{c}(x) d x=1 .
$$


By the Askey-Roy $q$-beta integral (1.2), we have

$$
\begin{aligned}
\int_{\mathbb{R}} \frac{2 e^{y(2 c-1)}}{\left(-q^{1-c} e^{2 y},-q^{c} e^{-2 y} ; q\right)_{\infty}} d x & =\int_{\mathbb{R}} \frac{e^{2 y c}+e^{2 y(c-1)}}{\left(-q^{1-c} e^{2 y},-q^{c} e^{-2 y} ; q\right)_{\infty}} d y \\
& =\frac{1}{2} \int_{0}^{\infty} \frac{t^{c-1}+t^{c-2}}{\left(-q^{1-c} t,-q^{c} / t ; q\right)_{\infty}} d t \\
& =q^{c(c-1)} \frac{\pi}{\sin \pi c} \frac{\left(q^{c}, q^{1-c} ; q\right)_{\infty}}{(q ; q)_{\infty}}
\end{aligned}
$$

because the integral

$$
\int_{0}^{\infty} \frac{t^{c-2}}{\left(-q^{1-c} t,-q^{c} / t ; q\right)_{\infty}} d t=\int_{0}^{\infty} \frac{s^{-c}}{\left(-q^{1-c} / s,-q^{c} s ; q\right)_{\infty}} d s
$$

is symmetric in $c$ and $1-c$. So $v_{c}$ is indeed the density of a probability measure.

Since $v_{c+1}=v_{c}$, it suffices to consider $v_{c}$ for $0<c \leq 1$. Besides the special cases $c=1$ and $c=1 / 2$, which lead to $\tilde{w}(x)$ and $w(x)$, the family of solutions presented in Corollary 5.3 are new. We note that the integral in (1.6) takes the form

$$
\begin{aligned}
& \int_{\mathbb{R}} \frac{e^{2 c y}+e^{2(c-1) y}}{\left(-q^{1-c} e^{2 y},-q^{c} e^{-2 y} ; q\right)_{\infty}} \prod_{j=1}^{4}\left(-t_{j} e^{y}, t_{j} e^{-y} ; q\right)_{\infty} d y \\
& \quad=q^{c(c-1)} \frac{\pi}{\sin \pi c} \frac{\left(q^{c}, q^{1-c} ; q\right)_{\infty}}{(q ; q)_{\infty}} \frac{\prod_{1 \leq j<k \leq 4}\left(-t_{j} t_{k} / q ; q\right)_{\infty}}{\left(t_{1} t_{2} t_{3} t_{4} / q^{3} ; q\right)_{\infty}}
\end{aligned}
$$

when $d \psi=v_{c}(x) d x$.

It is known that the Pick function being equal to the constant

$$
i \frac{\sqrt{c}}{2} \frac{\left(-1 / c,-c q^{2}, q ; q^{2}\right)_{\infty}}{\left(-q / c,-c q, q^{2} ; q^{2}\right)_{\infty}}
$$

in the open upper half-plane corresponds to the solution with density (5.4) in the Nevanlinna parametrization. Therefore, the Pick function corresponding to the density $w(x)$ equals the constant

$$
i \frac{3}{2 \sqrt{2}} \frac{\left(-2 q^{2},-q^{2} / 2, q ; q^{2}\right)_{\infty}}{\left(-2 q,-q / 2, q^{2} ; q^{2}\right)_{\infty}}
$$

in the open upper half-plane. It seems to be hard to determine the Pick functions corresponding to solutions from Corollary 5.3 when $c \neq 1 / 2$.

\section{Discrete solutions}

Recall from the proof of Theorem 5.1 that the orthogonality relation has the form

$$
\int_{\mathbb{R}} Q_{n}(x ; \beta) Q_{m}(x ; \beta) v(x ; \beta) d x=\frac{\beta^{n}(-1 / \beta ; p)_{n}}{p^{n}(p ; p)_{n}} \delta_{n, m} .
$$

According to Proposition 1.1 in [7] and the $q$-binomial theorem [10, II. 3], we thus have

$$
\begin{aligned}
\int_{\mathbb{R}} \sum_{n=0}^{\infty} Q_{n}(x ; \beta) t_{1}^{n} \sum_{m=0}^{\infty} Q_{m}(x ; \beta) t_{2}^{m} v(x ; \beta) d x & =\sum_{n=0}^{\infty} \frac{(-1 / \beta ; p)_{n}}{(p ; p)_{n}}\left(t_{1} t_{2} \beta / p\right)^{n} \\
& =\frac{\left(-t_{1} t_{2} / p ; p\right)_{\infty}}{\left(t_{1} t_{2} \beta / p ; p\right)_{\infty}}
\end{aligned}
$$


whenever $\left|t_{1}\right|,\left|t_{2}\right|<\sqrt{p / \beta}$. In view of (3.1) and since the right-hand side of (6.1) only depends on $t_{1} t_{2}$, a positive measure $\mu$ is solution to the moment problem if and only if

$$
\begin{aligned}
& \int_{\mathbb{R}}\left(t_{1} e^{-y},-t_{1} e^{y}, t_{2} e^{-y},-t_{2} e^{y} ; p\right)_{\infty} d \mu(x) \\
& \quad=\frac{\left(-t_{1} t_{2} / p ; p\right)_{\infty}}{\left(t_{1} t_{2} \beta / p ; p\right)_{\infty}}\left(-t_{1}^{2} \beta,-t_{2}^{2} \beta ; p^{2}\right)_{\infty}, \quad\left|t_{1} t_{2} \beta / p\right|<1
\end{aligned}
$$

For $a>0$ consider the discrete measure $\lambda_{a}^{(\beta)}$ supported on $\left\{x_{n}(a) \mid n \in \mathbb{Z}\right\}$ and defined by

$$
\lambda_{a}^{(\beta)}\left(\left\{x_{n}(a)\right\}\right)=\frac{\sqrt{1+x_{n}^{2}(a)}}{L(a)} \tilde{v}\left(x_{n}(a) ; \beta\right), \quad n \in \mathbb{Z}
$$

where

$$
\begin{gathered}
x_{n}(a):=x_{n}(a ; p)=\frac{1}{2}\left(\frac{1}{a p^{n}}-a p^{n}\right), \quad n \in \mathbb{Z}, \\
\tilde{v}(x ; \beta)=\frac{1}{\left(-e^{2 y} / \beta,-e^{-2 y} / \beta ; p^{2}\right)_{\infty}}, \quad x=\sinh y \in \mathbb{R},
\end{gathered}
$$

and $L(a)$ is a certain constant so that $\lambda_{a}^{(\beta)}$ becomes a probability measure. As we shall see below, these measures turn out to be discrete solutions to the moment problem. It is remarkable that they are constructed in the same way as one can obtain the $N$-extremal solutions to the $q^{-1}$-Hermite moment problem from the weight function in (1.3) or any other function from Theorem 5.2 .

Direct computations lead to

$$
\begin{aligned}
\int_{\mathbb{R}} & \left(t_{1} e^{-y},-t_{1} e^{y}, t_{2} e^{-y},-t_{2} e^{y} ; p\right)_{\infty} d \lambda_{a}^{(\beta)}(x) \\
= & \frac{1}{L(a)} \sum_{n=-\infty}^{\infty} \frac{1}{2}\left(\frac{1}{a p^{n}}+a p^{n}\right) \frac{\left(t_{1} a p^{n},-t_{1} / a p^{n}, t_{2} a p^{n},-t_{2} / a p^{n} ; p\right)_{\infty}}{\left(-1 / a^{2} p^{2 n} \beta,-a^{2} p^{2 n} / \beta ; p^{2}\right)_{\infty}} \\
= & \frac{1}{2 L(a)} \frac{\left(t_{1} a,-t_{1} / a, t_{2} a,-t_{2} / a ; p\right)_{\infty}}{\left(-1 / a^{2} \beta,-a^{2} / \beta ; p^{2}\right)_{\infty}} \\
& \times \sum_{n=-\infty}^{\infty}\left(1+a^{2} p^{2 n}\right) \frac{\left(-a^{2} / \beta ; p^{2}\right)_{n}}{\left(-1 / a^{2} p^{2 n} \beta ; p^{2}\right)_{n}} \frac{\left(-t_{1} / a p^{n},-t_{2} / a p^{n} ; p\right)_{n}}{\left(t_{1} a, t_{2} a ; p\right)_{n}} \frac{1}{a p^{n}}
\end{aligned}
$$

and due to Bailey's ${ }_{6} \psi_{6}$ sum [10, II. 33], we have

$$
\begin{aligned}
& \sum_{n=-\infty}^{\infty}\left(1+a^{2} p^{2 n}\right) \frac{\left(-a^{2} / \beta ; p^{2}\right)_{n}}{\left(-1 / a^{2} p^{2 n} \beta ; p^{2}\right)_{n}} \frac{\left(-t_{1} / a p^{n},-t_{2} / a p^{n} ; p\right)_{n}}{\left(t_{1} a, t_{2} a ; p\right)_{n}} \frac{1}{a p^{n}} \\
& =\frac{1+a^{2}}{a} \sum_{n=-\infty}^{\infty} \frac{\left(-a^{2} p^{2},-a^{2} / \beta ; p^{2}\right)_{n}}{\left(-a^{2},-a^{2} p^{2} \beta ; p^{2}\right)_{n}} \frac{\left(-a p / t_{1},-a p / t_{2} ; p\right)_{n}}{\left(t_{1} a, t_{2} a ; p\right)_{n}}\left(t_{1} t_{2} \beta / p\right)^{n} \\
& =\frac{1+a^{2}}{a} \frac{\left(-a^{2} p,-p / a^{2},-p \beta,-t_{1} t_{2} / p, p ; p\right)_{\infty}}{\left(t_{1} a,-t_{1} / a, t_{2} a,-t_{2} / a, t_{1} t_{2} \beta / p ; p\right)_{\infty}} \frac{\left(-t_{1}^{2} \beta,-t_{2}^{2} \beta ; p^{2}\right)_{\infty}}{\left(-a^{2} p^{2} \beta,-p^{2} \beta / a^{2} ; p^{2}\right)_{\infty}}
\end{aligned}
$$


for $\left|t_{1} t_{2} \beta / p\right|<1$. Consequently,

$$
\begin{aligned}
\int_{\mathbb{R}} & \left(t_{1} e^{-y},-t_{1} e^{y}, t_{2} e^{-y},-t_{2} e^{y} ; p\right)_{\infty} d \lambda_{a}^{(\beta)}(x) \\
= & \frac{1+a^{2}}{2 a L(a)} \frac{\left(-a^{2} p,-p / a^{2},-p \beta, p ; p\right)_{\infty}}{\left(-a^{2} / \beta,-1 / a^{2} \beta,-a^{2} p^{2} \beta,-p^{2} \beta / a^{2} ; p^{2}\right)_{\infty}} \\
& \times \frac{\left(-t_{1} t_{2} / \beta ; p\right)_{\infty}}{\left(t_{1} t_{2} \beta / p ; p\right)_{\infty}}\left(-t_{1}^{2} \beta,-t_{2}^{2} \beta ; p^{2}\right)_{\infty}
\end{aligned}
$$

and with

$$
L(a)=\frac{1+a^{2}}{2 a} \frac{\left(-a^{2} p,-p / a^{2},-p \beta, p ; p\right)_{\infty}}{\left(-a^{2} / \beta,-1 / a^{2} \beta,-a^{2} p^{2} \beta,-p^{2} \beta / a^{2} ; p^{2}\right)_{\infty}},
$$

the following result is obtained.

Theorem 6.1. The discrete measures

$$
\lambda_{a}^{(\beta)}=\frac{\left(-a^{2} p^{2} \beta,-p^{2} \beta / a^{2} ; p^{2}\right)_{\infty}}{\left(-a^{2},-p / a^{2},-p \beta, p ; p\right)_{\infty}} \sum_{n=-\infty}^{\infty} \frac{\left(-a^{2} / \beta ; p^{2}\right)_{n}}{\left(-a^{2} p^{2} \beta ; p^{2}\right)_{n}} a^{2 n} \beta^{n}\left(1+a^{2} p^{2 n}\right) p^{n^{2}} \varepsilon_{x_{n}(a)}
$$

are solutions to the moment problem.

Since $\lambda_{a p}^{(\beta)}=\lambda_{a}^{(\beta)}$, it suffices to consider $p<a \leq 1$. In the special case $\beta=0$, the measures in Theorem 6.1 reduce to

$$
\nu_{a}=\frac{1}{\left(-a^{2},-q / a^{2}, q ; q\right)_{\infty}} \sum_{n=-\infty}^{\infty} a^{4 n}\left(1+a^{2} q^{2 n}\right) q^{n(2 n-1)} \varepsilon_{x_{n}(a ; q)}
$$

if we replace $p$ by $q$. That is, we obtain the $N$-extremal solutions to the $q^{-1}$ Hermite moment problem. So for a moment one may believe that the solutions $\lambda_{a}^{(\beta)}$ are $N$-extremal. However, the special case $\beta=1 / p$ reads

$$
\tilde{\nu}_{a}=\frac{1}{2\left(-a^{2},-p^{2} / a^{2}, p^{2} ; p^{2}\right)_{\infty}} \sum_{n=-\infty}^{\infty} a^{2 n}\left(1+a^{2} p^{2 n}\right) p^{n^{2}-n} \varepsilon_{x_{n}(a ; p)}
$$

and since

$$
\begin{aligned}
\sum_{n=-\infty}^{\infty} a^{2 n}\left(1+a^{2} p^{2 n}\right) p^{n^{2}-n} \varepsilon_{x_{n}(a ; p)}= & \sum_{n=-\infty}^{\infty} a^{4 n}\left(1+a^{2} p^{4 n}\right) p^{4 n^{2}-2 n} \varepsilon_{x_{n}\left(a ; p^{2}\right)} \\
& +\sum_{n=-\infty}^{\infty} a^{4 n+2}\left(1+a^{2} p^{4 n+2}\right) p^{4 n^{2}+2 n} \varepsilon_{x_{n}\left(a p ; p^{2}\right)}
\end{aligned}
$$

we see after replacing $p^{2}$ with $q$ that

$$
\tilde{\nu}_{a}=\frac{1}{2}\left(\nu_{a}+\nu_{a \sqrt{q}}\right),
$$

where $\nu_{a}$ is defined in (6.3) (or (1.7)). It is plausible that the supports of the $N$-extremal solutions in some way should depend on $\beta$. 


\section{A BILINEAR GENERATING FUNCTION}

In this section we shall derive a bilinear generating function for the polynomials $Q_{n}(x ; \beta)$. In particular, an expression for the Poisson kernel will be obtained. We follow more or less the same procedure as Ismail and Stanton in [15] and [16. Unless otherwise stated, it is assumed that $\beta>0$.

Lemma 7.1. The polynomials $Q_{n}(x ; \beta)$ have the $p$-integral representation

$$
\begin{aligned}
(-1)^{n} p^{n} \frac{(p ; p)_{n}}{(-1 / \beta ; p)_{n}} Q_{n}(x ; \beta)= & \frac{\left(-e^{2 y} / \beta,-e^{-2 y} / \beta ; p^{2}\right)_{\infty}}{2 i p \sqrt{\beta}(-1 / \beta ; p)_{\infty}} \frac{\left(p ; p^{2}\right)_{\infty}}{\left(p^{2} ; p^{2}\right)_{\infty}} \\
& \times \frac{1}{1-p} \int_{-i p \sqrt{\beta}}^{i p \sqrt{\beta}} t^{n} \frac{(i t / \sqrt{\beta},-i t / \sqrt{\beta} ; p)_{\infty}}{\left(t e^{y} / p \beta,-t e^{-y} / p \beta ; p\right)_{\infty}} d_{p} t .
\end{aligned}
$$

Proof. By definition of the $p$-integral, we have

$$
\begin{aligned}
& \frac{1}{1-p} \int_{-i p \sqrt{\beta}}^{i p \sqrt{\beta}} t^{n} \frac{(i t / \sqrt{\beta},-i t / \sqrt{\beta} ; p)_{\infty}}{\left(t e^{y} / p \beta,-t e^{-y} / p \beta ; p\right)_{\infty}} d_{p} t \\
& =(i p \sqrt{\beta})^{n+1} \sum_{k=0}^{\infty} \frac{\left(-p^{k+1}, p^{k+1} ; p\right)_{\infty}}{\left(i p^{k} e^{y} / \sqrt{\beta},-i p^{k} e^{-y} / \sqrt{\beta} ; p\right)_{\infty}}\left(p^{n+1}\right)^{k} \\
& -(-i p \sqrt{\beta})^{n+1} \sum_{k=0}^{\infty} \frac{\left(p^{k+1},-p^{k+1} ; p\right)_{\infty}}{\left(-i p^{k} e^{y} / \sqrt{\beta}, i p^{k} e^{-y} / \sqrt{\beta} ; p\right)_{\infty}}\left(p^{n+1}\right)^{k} \\
& =(i p \sqrt{\beta})^{n+1}\left\{\frac{\left(p^{2} ; p^{2}\right)_{\infty}}{\left(i e^{y} / \sqrt{\beta},-i e^{-y} / \sqrt{\beta} ; p\right)_{\infty}}{ }_{2} \phi_{1}\left(\begin{array}{c}
i e^{y} / \sqrt{\beta},-i e^{-y} / \sqrt{\beta} \\
-p
\end{array} \mid p, p^{n+1}\right)\right. \\
& +(-1)^{n} \frac{\left(p^{2} ; p^{2}\right)_{\infty}}{\left(-i e^{y} / \sqrt{\beta}, i e^{-y} / \sqrt{\beta} ; p\right)_{\infty}}{ }_{2} \phi_{1}\left(\begin{array}{c|c}
-i e^{y} / \sqrt{\beta}, i e^{-y} / \sqrt{\beta} & p, p^{n+1} \\
-p &
\end{array}\right\} \\
& =(-1)^{n} \frac{(i p \sqrt{\beta})^{n+1}\left(p^{2} ; p^{2}\right)_{\infty}}{\left(-i e^{y} / \sqrt{\beta}, i e^{-y} / \sqrt{\beta} ; p\right)_{\infty}}\left\{{ }_{2} \phi_{1}\left(\begin{array}{c}
i e^{-y} / \sqrt{\beta},-i e^{y} / \sqrt{\beta} \\
-p
\end{array} \mid p, p^{n+1}\right)\right. \\
& \left.+(-1)^{n} \frac{\left(-i e^{y} / \sqrt{\beta}, i e^{-y} / \sqrt{\beta} ; p\right)_{\infty}}{\left(i e^{y} / \sqrt{\beta},-i e^{-y} / \sqrt{\beta} ; p\right)_{\infty}} \phi_{1}\left(\begin{array}{c}
-i e^{-y} / \sqrt{\beta}, i e^{y} / \sqrt{\beta} \\
-p
\end{array} \mid p, p^{n+1}\right)\right\} .
\end{aligned}
$$

According to [10, III. 31], the combination of ${ }_{2} \phi_{1}$ 's in the bracket reduces to

$$
\begin{aligned}
\frac{\left(-p^{n} / \beta,-1 ; p\right)_{\infty}}{\left(-i p^{n} e^{-y} / \sqrt{\beta},-i p e^{y} \sqrt{\beta} ; p\right)_{\infty}}{ }_{2} \phi_{1}\left(\begin{array}{c}
i p e^{y} \sqrt{\beta},-p^{1-n} \beta \\
i e^{y} p^{1-n} \sqrt{\beta}
\end{array} \mid p, i e^{y} / \sqrt{\beta}\right) \\
=\frac{2(-1 / \beta,-p ; p)_{\infty}}{\left(-i e^{-y} / \sqrt{\beta},-i p e^{y} \sqrt{\beta} ; p\right)_{\infty}} \frac{\left(-i e^{-y} / \sqrt{\beta} ; p\right)_{n}}{(-1 / \beta ; p)_{n}} \\
\quad \times_{2} \phi_{1}\left(\begin{array}{c}
i p e^{y} \sqrt{\beta},-p^{1-n} \beta \\
i e^{y} p^{1-n} \sqrt{\beta}
\end{array} \mid p, i e^{y} / \sqrt{\beta}\right),
\end{aligned}
$$

and by Heine's transformation formula [10, III. 3], the above ${ }_{2} \phi_{1}$ can be written as

$$
\frac{\left(-i p e^{y} \sqrt{\beta} ; p\right)_{\infty}}{\left(i e^{y} / \sqrt{\beta} ; p\right)_{\infty}} \phi_{1}\left(\begin{array}{c}
p^{-n},-i e^{y} / \sqrt{\beta} \\
i e^{y} p^{1-n} \sqrt{\beta}
\end{array} \mid p,-i p e^{y} \sqrt{\beta}\right) .
$$

Recalling the explicit form of the polynomials $Q_{n}(x ; \beta)$, the representation follows easily. 
Theorem 7.2. For $|z|<1$, the polynomials $Q_{n}(x ; \beta)$ satisfy the bilinear generating function

$$
\begin{aligned}
\sum_{n=0}^{\infty} & \frac{(p ; p)_{n}}{(-1 / \beta ; p)_{n}} Q_{n}(x ; \beta) Q_{n}\left(x^{\prime} ; \beta\right)(z / \beta)^{n} \\
= & \frac{\left(-i e^{y} / \sqrt{\beta}, i e^{-y} / \sqrt{\beta}, i z e^{y^{\prime}} / \sqrt{\beta},-i z e^{-y^{\prime}} / \sqrt{\beta} ; p\right)_{\infty}}{2(-1 / \beta ; p)_{\infty}} \\
& \times \frac{\left(p ; p^{2}\right)_{\infty}}{\left(z^{2} ; p^{2}\right)_{\infty}} \phi_{3}\left(\begin{array}{c}
i e^{y} / \sqrt{\beta},-i e^{-y} / \sqrt{\beta}, z,-z \\
i z e^{y^{\prime}} / \sqrt{\beta},-i z e^{-y^{\prime}} / \sqrt{\beta},-p
\end{array} \mid p, p\right) \\
& + \text { a similar term with y replaced by }-y \text { and } y^{\prime} \text { replaced by }-y^{\prime} .
\end{aligned}
$$

Proof. By the previous lemma, we have

$$
\begin{aligned}
\sum_{n=0}^{\infty} & \frac{(p ; p)_{n}}{(-1 / \beta ; p)_{n}} Q_{n}(x ; \beta) Q_{n}\left(x^{\prime} ; \beta\right)(z / \beta)^{n} \\
= & \frac{\left(-e^{2 y} / \beta,-e^{-2 y} / \beta ; p^{2}\right)_{\infty}}{2 i p \sqrt{\beta}(-1 / \beta ; p)_{\infty}} \frac{\left(p ; p^{2}\right)_{\infty}}{\left(p^{2} ; p^{2}\right)_{\infty}} \sum_{n=0}^{\infty} Q_{n}\left(x^{\prime} ; \beta\right)(-z / p \beta)^{n} \\
& \times \frac{1}{1-p} \int_{-i p \sqrt{\beta}}^{i p \sqrt{\beta}} t^{n} \frac{(i t / \sqrt{\beta},-i t / \sqrt{\beta} ; p)_{\infty}}{\left(t e^{y} / p \beta,-t e^{-y} / p \beta ; p\right)_{\infty}} d_{p} t .
\end{aligned}
$$

Interchanging the order of summation and integration, the above sum reduces to

$$
\begin{aligned}
& \frac{1}{1-p} \int_{-i p \sqrt{\beta}}^{i p \sqrt{\beta}} \frac{(i t / \sqrt{\beta},-i t / \sqrt{\beta} ; p)_{\infty}}{\left(t e^{y} / p \beta,-t e^{-y} / p \beta ; p\right)_{\infty}} \sum_{n=0}^{\infty} Q_{n}\left(x^{\prime} ; \beta\right)(-z t / p \beta)^{n} d_{p} t \\
& =\frac{1}{1-p} \int_{-i p \sqrt{\beta}}^{i p \sqrt{\beta}} \frac{\left(i t / \sqrt{\beta},-i t / \sqrt{\beta}, z t e^{y^{\prime}} / p \beta,-z t e^{-y^{\prime}} / p \beta ; p\right)_{\infty}}{\left(t e^{y} / p \beta,-t e^{-y} / p \beta, i z t / p \sqrt{\beta},-i z t / p \sqrt{\beta} ; p\right)_{\infty}} d_{p} t
\end{aligned}
$$

for $|z t / p|<\sqrt{\beta}$. Here the $p$-integral can be written as

$$
\begin{aligned}
i p & \sqrt{\beta} \sum_{n=0}^{\infty} \frac{\left(-p^{n+1}, p^{n+1}, i z p^{n} e^{y^{\prime}} / \sqrt{\beta},-i z p^{n} e^{-y^{\prime}} / \sqrt{\beta} ; p\right)_{\infty}}{\left(i p^{n} e^{y} / \sqrt{\beta},-i p^{n} e^{-y} / \sqrt{\beta},-z p^{n}, z p^{n} ; p\right)_{\infty}} p^{n} \\
+i p & \sqrt{\beta} \sum_{n=0}^{\infty} \frac{\left(p^{n+1},-p^{n+1},-i z p^{n} e^{y^{\prime}} / \sqrt{\beta}, i z p^{n} e^{-y^{\prime}} / \sqrt{\beta} ; p\right)_{\infty}}{\left(-i p^{n} e^{y} / \sqrt{\beta}, i p^{n} e^{-y} / \sqrt{\beta}, z p^{n},-z p^{n} ; p\right)_{\infty}} p^{n} \\
= & i p \sqrt{\beta} \frac{\left(p^{2} ; p^{2}\right)_{\infty}}{\left(i z e^{y^{\prime}} / \sqrt{\beta},-i z e^{-y^{\prime}} / \sqrt{\beta} ; p\right)_{\infty}} \\
& \times_{4} \phi_{3}\left(\begin{array}{c}
i e^{y} / \sqrt{\beta},-i e^{-y} / \sqrt{\beta}, z,-z \\
i z e^{y^{\prime}} / \sqrt{\beta},-i z e^{-y^{\prime}} / \sqrt{\beta},-p
\end{array} \mid p, p\right)
\end{aligned}
$$

+ a similar term with $y$ replaced by $-y$ and $y^{\prime}$ replaced by $-y^{\prime}$,

and the theorem is proved. 
Corollary 7.3. For $|z|<1 / p$, the Poisson kernel is given by

$$
\begin{aligned}
\sum_{n=0}^{\infty} P_{n}(x ; \beta) P_{n}\left(x^{\prime} ; \beta\right) z^{n}= & \frac{\left(-i e^{y} / \sqrt{\beta}, i e^{-y} / \sqrt{\beta}, i z p e^{y^{\prime}} / \sqrt{\beta},-i z p e^{-y^{\prime}} / \sqrt{\beta} ; p\right)_{\infty}}{2(-1 / \beta ; p)_{\infty}} \\
& \times \frac{\left(p ; p^{2}\right)_{\infty}}{\left(z^{2} p^{2} ; p^{2}\right)_{\infty}}{ }_{4} \phi_{3}\left(\begin{array}{c}
i e^{y} / \sqrt{\beta},-i e^{-y} / \sqrt{\beta}, z p,-z p \\
i z p e^{y^{\prime}} / \sqrt{\beta},-i z p e^{-y^{\prime}} / \sqrt{\beta},-p
\end{array} \mid p, p\right) \\
& + \text { a similar term with } y \text { and } y^{\prime} \text { replaced by }-y \text { and }-y^{\prime} .
\end{aligned}
$$

In particular, we have

$$
\begin{aligned}
\sum_{n=0}^{\infty} P_{n}^{2}(x ; \beta)= & \frac{\left(-e^{2 y} / \beta,-e^{-2 y} / \beta ; p^{2}\right)_{\infty}}{(-1 / \beta ; p)_{\infty}} \frac{\left(p ; p^{2}\right)_{\infty}}{\left(p^{2} ; p^{2}\right)_{\infty}} \\
& \times \sum_{n=0}^{\infty} \frac{1+p^{2 n} / \beta}{\left(1+p^{2 n} e^{2 y} / \beta\right)\left(1+p^{2 n} e^{-2 y} / \beta\right)} p^{n} .
\end{aligned}
$$

Proof. The first part follows immediately from (5.2). With $z=1$ and $x^{\prime}=x$, the Poisson kernel reduces to

$$
\begin{aligned}
& \frac{\left(-e^{2 y} / \beta,-e^{-2 y} / \beta ; p^{2}\right)_{\infty}}{2(-1 / \beta ; p)_{\infty}} \frac{\left(p ; p^{2}\right)_{\infty}}{\left(p^{2} ; p^{2}\right)_{\infty}} \\
& \times \sum_{n=0}^{\infty}\left\{\frac{p^{n}}{\left(1-i p^{n} e^{y} / \sqrt{\beta}\right)\left(1+i p^{n} e^{-y} / \sqrt{\beta}\right)}+\frac{p^{n}}{\left(1+i p^{n} e^{y} / \sqrt{\beta}\right)\left(1-i p^{n} e^{-y} / \sqrt{\beta}\right)}\right\} \\
& \quad=\frac{\left(-e^{2 y} / \beta,-e^{-2 y} / \beta ; p^{2}\right)_{\infty}}{(-1 / \beta ; p)_{\infty}} \frac{\left(p ; p^{2}\right)_{\infty}}{\left(p^{2} ; p^{2}\right)_{\infty}} \sum_{n=0}^{\infty} \frac{1+p^{2 n} / \beta}{\left(1+p^{2 n} e^{2 y} / \beta\right)\left(1+p^{2 n} e^{-2 y} / \beta\right)} p^{n}
\end{aligned}
$$

and this proves the second part.

With an explicit expression for the Poisson kernel at hand one should be able to explain that the discrete solutions $\lambda_{a}^{(\beta)}$ in Theorem 6.1 are not $N$-extremal. Recall that the masses of the $N$-extremal solutions are given by the function

$$
\rho(x ; \beta)=\left(\sum_{n=0}^{\infty} P_{n}^{2}(x ; \beta)\right)^{-1}, \quad x \in \mathbb{R} .
$$

When $x=0$, the value is

$$
\rho(0 ; \beta)=\frac{\left(p^{2} ; p^{2}\right)_{\infty}}{\left(p ; p^{2}\right)_{\infty}} \frac{\left(-p / \beta ; p^{2}\right)_{\infty}}{\left(-1 / \beta ; p^{2}\right)_{\infty}}\left(\sum_{n=0}^{\infty} \frac{p^{n}}{1+p^{2 n} / \beta}\right)^{-1},
$$

and hence

$$
\begin{aligned}
& \frac{1}{\rho(0 ; \beta)}+\frac{1}{\rho(0 ; 1 / \beta)} \\
& \quad=\frac{\left(p ; p^{2}\right)_{\infty}}{\left(p^{2} ; p^{2}\right)_{\infty}}\left(\frac{\left(-1 / \beta ; p^{2}\right)_{\infty}}{\left(-p / \beta ; p^{2}\right)_{\infty}} \sum_{n=0}^{\infty} \frac{p^{n}}{1+p^{2 n} / \beta}+\frac{\left(-\beta ; p^{2}\right)_{\infty}}{\left(-p \beta ; p^{2}\right)_{\infty}} \sum_{n=0}^{\infty} \frac{p^{n}}{1+p^{2 n} \beta}\right) .
\end{aligned}
$$

By Ramanujan's ${ }_{1} \psi_{1}$ sum [10, II. 29], we have

$$
\sum_{n=-\infty}^{\infty} \frac{\left(-1 / \beta ; p^{2}\right)_{n}}{\left(-p^{2} / \beta ; p^{2}\right)_{n}} p^{n}=\frac{\left(p^{2} ; p^{2}\right)_{\infty}^{2}}{\left(p ; p^{2}\right)_{\infty}^{2}} \frac{\left(-p / \beta,-p \beta ; p^{2}\right)_{\infty}}{\left(-p^{2} / \beta,-p^{2} \beta ; p^{2}\right)_{\infty}}
$$


and thus

$$
\begin{gathered}
\frac{\left(-p / \beta ; p^{2}\right)_{\infty}}{\left(-p^{2} / \beta ; p^{2}\right)_{\infty}} \frac{1}{\rho(0 ; \beta)}+\frac{\left(-p \beta ; p^{2}\right)_{\infty}}{\left(-p^{2} \beta ; p^{2}\right)_{\infty}} \frac{1}{\rho(0 ; 1 / \beta)} \\
\quad=\frac{\left(p ; p^{2}\right)_{\infty}}{\left(p^{2} ; p^{2}\right)_{\infty}}\left(1+\frac{\left(p^{2} ; p^{2}\right)_{\infty}^{2}}{\left(p ; p^{2}\right)_{\infty}^{2}} \frac{\left(-p / \beta,-p \beta ; p^{2}\right)_{\infty}}{\left(-p^{2} / \beta,-p^{2} \beta ; p^{2}\right)_{\infty}}\right) \\
\quad=\frac{\left(p ; p^{2}\right)_{\infty}}{\left(p^{2} ; p^{2}\right)_{\infty}}+\frac{\left(p^{2} ; p^{2}\right)_{\infty}}{\left(p ; p^{2}\right)_{\infty}} \frac{\left(-p / \beta,-p \beta ; p^{2}\right)_{\infty}}{\left(-p^{2} / \beta,-p^{2} \beta ; p^{2}\right)_{\infty}} .
\end{gathered}
$$

On the other hand,

$$
\lambda_{1}^{(\beta)}(\{0\})=\frac{\left(p ; p^{2}\right)_{\infty}}{\left(p^{2} ; p^{2}\right)_{\infty}} \frac{\left(-p^{2} \beta ; p^{2}\right)_{\infty}}{\left(-p \beta ; p^{2}\right)_{\infty}}
$$

so that

$$
\begin{gathered}
\frac{\left(-p / \beta ; p^{2}\right)_{\infty}}{\left(-p^{2} / \beta ; p^{2}\right)_{\infty}} \frac{1}{\lambda_{1}^{(\beta)}(\{0\})}+\frac{\left(-p \beta ; p^{2}\right)_{\infty}}{\left(-p^{2} \beta ; p^{2}\right)_{\infty}} \frac{1}{\lambda_{1}^{(1 / \beta)}(\{0\})} \\
\quad=2 \frac{\left(p^{2} ; p^{2}\right)_{\infty}}{\left(p ; p^{2}\right)_{\infty}} \frac{\left(-p / \beta,-p \beta ; p^{2}\right)_{\infty}}{\left(-p^{2} / \beta,-p^{2} \beta ; p^{2}\right)_{\infty}} .
\end{gathered}
$$

Since

$$
\sum_{n=-\infty}^{\infty} \frac{\left(-1 / \beta ; p^{2}\right)_{n}}{\left(-p^{2} / \beta ; p^{2}\right)_{n}} p^{n}>1
$$

we get from (7.1) that

$$
\frac{\left(-p / \beta,-p \beta ; p^{2}\right)_{\infty}}{\left(-p^{2} / \beta,-p^{2} \beta ; p^{2}\right)_{\infty}}>\frac{\left(p ; p^{2}\right)_{\infty}^{2}}{\left(p^{2} ; p^{2}\right)_{\infty}^{2}}
$$

and as a consequence, the expression in (7.2) is $<$ the expression in (7.3). So for each $\beta>0$, we either have

$$
\rho(0 ; \beta)>\lambda_{1}^{(\beta)}(\{0\})
$$

or

$$
\rho(0 ; 1 / \beta)>\lambda_{1}^{(1 / \beta)}(\{0\}) .
$$

In particular, this means that $\rho(0 ; 1)>\lambda_{1}^{(1)}(\{0\})$ and at least when $\beta=1$, the solution $\lambda_{1}^{(\beta)}$ is not $N$-extremal.

\section{Some Biorthogonal Rational FUnCtions}

In [7. Berg and Ismail have shown how to systematically build the classical $q$ orthogonal polynomials from the $q$-Hermite polynomials using a simple procedure of attaching generating functions to measures. As an example, the attachment procedure for the $q^{-1}$-Hermite polynomials leads to the polynomials

$$
u_{n}\left(x ; t_{1}, t_{2}\right)=\frac{\left(-q e^{-y} / t_{2} ; q\right)_{n}}{(q ; q)_{n}}\left(-t_{2} / q\right)^{n}{ }_{2} \phi_{1}\left(\begin{array}{c}
q^{-n}, q e^{y} / t_{1} \\
-t_{2} e^{y} / q^{n}
\end{array} \mid q,-t_{1} e^{y}\right)
$$

which are special cases of the Al-Salam-Chihara polynomials $v_{n}(x)$ (corresponding to $q>1$ ) from [4. If we set $t_{1}=-t_{2}=i q \sqrt{\beta}$ for some $\beta \geq 0$ and replace $q$ by $p$, the polynomials in (8.1) reduce to $Q_{n}(x ; \beta)$. At the second stage, the attachment 
procedure is applied to $\left\{u_{n}\right\}$ and leads to the biorthogonal rational functions

$$
\varphi_{n}\left(x ; t_{1}, t_{2}, t_{3}, t_{4}\right)={ }_{4} \phi_{3}\left(\begin{array}{c|c}
q^{-n},-t_{1} t_{2} q^{n-2},-t_{1} t_{3} / q,-t_{1} t_{4} / q \\
-t_{1} e^{y}, t_{1} e^{-y}, t_{1} t_{2} t_{3} t_{4} / q^{3}
\end{array} \mid q, q\right)
$$

studied by Ismail and Masson in [13.

It is known that the rational functions $\varphi_{n}\left(x ; t_{1}, t_{2}, t_{3}, t_{4}\right)$ are biorthogonal with respect to any measure $\mu$ of the form

$$
d \mu(x)=\prod_{j=1}^{4}\left(t_{j} e^{-y},-t_{j} e^{y} ; q\right)_{\infty} d \psi(x),
$$

where $\psi$ is a solution to the $q^{-1}$-Hermite moment problem. In this section we show how solutions to the moment problem associated with the polynomials $Q_{n}(x ; \beta)$ lead to biorthogonality relations for the special case $t_{3}=-t_{4}=i q \sqrt{\beta}$ of the rational functions in (8.2).

Theorem 8.1. Suppose that $\nu$ is a positive measure such that

$$
\int_{\mathbb{R}} Q_{n}(x ; \beta) Q_{m}(x ; \beta) d \nu(x)=\frac{\beta^{n}(-1 / \beta ; p)_{n}}{p^{n}(p ; p)_{n}} \delta_{n, m} .
$$

Then the rational functions

$$
\varphi_{n}\left(x ; t_{1}, t_{2}, \beta\right)={ }_{4} \phi_{3}\left(\begin{array}{c|c}
p^{-n}, i t_{1} \sqrt{\beta},-i t_{1} \sqrt{\beta},-t_{1} t_{2} p^{n-2} \\
-t_{1} e^{y}, t_{1} e^{-y}, t_{1} t_{2} \beta / p
\end{array} \mid p, p\right)
$$

are biorthogonal with respect to the measure $\mu$ given by

$$
d \mu(x)=\prod_{j=1}^{2}\left(t_{j} e^{-y},-t_{j} e^{y} ; q\right)_{\infty} d \nu(x),
$$

and the biorthogonality relation is

$$
\begin{aligned}
\int_{\mathbb{R}} \varphi_{n}\left(x ; t_{1}, t_{2}, \beta\right) \varphi_{m}\left(x ; t_{2}, t_{1}, \beta\right) d \mu(x)= & \frac{1+t_{1} t_{2} p^{n-2}}{1+t_{1} t_{2} p^{2 n-2}} \frac{(-1 / \beta, p ; p)_{n}\left(t_{1} t_{2} \beta / p\right)^{n}}{\left(t_{1} t_{2} \beta / p ; p\right)_{n}} \\
& \times \frac{\left(-t_{1} t_{2} p^{n-1} ; p\right)_{\infty}\left(-t_{1}^{2} \beta,-t_{2}^{2} \beta ; p^{2}\right)_{\infty}}{\left(t_{1} t_{2} \beta / p ; p\right)_{\infty}} \delta_{n, m} .
\end{aligned}
$$

Proof. To show the biorthogonality, it is sufficient to prove that

$$
\int_{\mathbb{R}} \frac{\varphi_{n}\left(x ; t_{1}, t_{2}, \beta\right)}{\left(t_{2} e^{-y},-t_{2} e^{y} ; p\right)_{m}} d \mu(x)=0 \quad \text { for } \quad 0 \leq m<n .
$$

According to (6.2), we have

$$
\begin{gathered}
\int_{\mathbb{R}}\left(t_{1} p^{k} e^{-y},-t_{1} p^{k} e^{y}, t_{2} p^{m} e^{-y},-t_{2} p^{m} e^{y} ; p\right)_{\infty} d \nu(x) \\
=\frac{\left(-t_{1} t_{2} p^{k+m-1} ; p\right)_{\infty}}{\left(t_{1} t_{2} \beta p^{k+m-1} ; p\right)_{\infty}}\left(-t_{1}^{2} \beta p^{2 k},-t_{2}^{2} \beta p^{2 m} ; p^{2}\right)_{\infty}
\end{gathered}
$$


and thus

$$
\begin{aligned}
\int_{\mathbb{R}} \frac{\varphi_{n}\left(x ; t_{1}, t_{2}, \beta\right)}{\left(t_{2} e^{-y},-t_{2} e^{y} ; p\right)_{m}} d \mu(x)= & \sum_{k=0}^{n} \frac{\left(p^{-n}, i t_{1} \sqrt{\beta},-i t_{1} \sqrt{\beta},-t_{1} t_{2} p^{n-2} ; p\right)_{k}}{\left(t_{1} t_{2} \beta / p, p ; p\right)_{k}} p^{k} \\
& \times \int_{\mathbb{R}}\left(t_{1} p^{k} e^{-y},-t_{1} p^{k} e^{y}, t_{2} p^{m} e^{-y},-t_{2} p^{m} e^{y} ; p\right)_{\infty} d \nu(x) \\
= & \frac{\left(-t_{1} t_{2} p^{m-1} ; p\right)_{\infty}}{\left(t_{1} t_{2} \beta p^{m-1} ; p\right)_{\infty}}\left(-t_{1}^{2} \beta,-t_{2}^{2} \beta p^{2 m} ; p^{2}\right)_{\infty} \\
& \times \sum_{k=0}^{n} \frac{\left(p^{-n},-t_{1} t_{2} p^{n-2}, t_{1} t_{2} \beta p^{m-1} ; p\right)_{k}}{\left(t_{1} t_{2} \beta / p,-t_{1} t_{2} p^{m-1}, p ; p\right)_{k}} p^{k} .
\end{aligned}
$$

By the $q$-Saalschütz sum [10, II. 12], the above sum is equal to

$$
\frac{\left(p^{m-n+1},-1 / \beta ; p\right)_{n}}{\left(-t_{1} t_{2} p^{m-1}, p^{2-n} / t_{1} t_{2} \beta ; p\right)_{n}}
$$

and

$$
\left(p^{m-n+1} ; p\right)_{n}=0 \quad \text { for } \quad 0 \leq m<n .
$$

The case $m=n$ reads

$$
\begin{aligned}
\int_{\mathbb{R}} \frac{\varphi_{n}\left(x ; t_{1}, t_{2}, \beta\right)}{\left(t_{2} e^{-y},-t_{2} e^{y} ; p\right)_{n}} d \mu(x)= & \frac{(p,-1 / \beta ; p)_{n}}{\left(-t_{1} t_{2} p^{n-1}, p^{2-n} / t_{1} t_{2} \beta ; p\right)_{n}} \\
& \times \frac{\left(-t_{1} t_{2} p^{n-1} ; p\right)_{\infty}}{\left(t_{1} t_{2} \beta p^{n-1} ; p\right)_{\infty}}\left(-t_{1}^{2} \beta,-t_{2}^{2} \beta p^{2 n} ; p^{2}\right)_{\infty},
\end{aligned}
$$

and the biorthogonality relation is established after multiplication by

$$
\frac{\left(p^{-n}, i t_{2} \sqrt{\beta},-i t_{2} \sqrt{\beta},-t_{1} t_{2} p^{n-2} ; p\right)_{n}}{\left(t_{1} t_{2} \beta / p, p ; p\right)_{n}} p^{n} .
$$

The special cases $d \nu=v(x ; \beta) d x$ and $\nu=\lambda_{a}^{(\beta)}$ are not leading to new measures of biorthogonality for the rational functions $\varphi_{n}\left(x ; t_{1}, t_{2}, t_{3}, t_{4}\right)$. In the first case, $\mu$ is of the form (8.3) with $d \psi=w_{\beta}(x) d x$, see (5.4), and in the second case, a more general result without the restrictions on $t_{3}$ and $t_{4}$ is contained in Theorem 4.2 in [13.

\section{A $p$-Sturm-Liouville equation}

The main result in this section is the $p$-Sturm-Liouville equation in Theorem 9.3 , As an application, we give an easy proof of the fact that the polynomials $Q_{n}(x ; \beta)$ are orthogonal with respect to the weight function

$$
\tilde{v}(x ; \beta)=\frac{1}{\left(-e^{2 y} / \beta,-e^{-2 y} / \beta ; p^{2}\right)_{\infty}}, \quad x=\sinh y \in \mathbb{R},
$$

and the discrete measures

$$
\tilde{\lambda}_{a}^{(\beta)}=\sum_{n=-\infty}^{\infty} \tilde{v}\left(x_{n}(a) ; \beta\right) \sqrt{1+x_{n}^{2}(a)} \varepsilon_{x_{n}(a)},
$$

where

$$
x_{n}(a)=\frac{1}{2}\left(\frac{1}{a p^{n}}-a p^{n}\right) .
$$


But we also use the $p$-Sturm-Liouville equation to derive a Bethe Ansatz-type relation satisfied by the zeros of $Q_{n}(x ; \beta)$.

The first step is to establish a lowering operator.

Lemma 9.1. A lowering operator for $Q_{n}(x ; \beta)$ is given by

$$
\mathcal{D}_{p} Q_{n+1}(x ; \beta)=\frac{2 p^{n / 2}}{1-p} Q_{n}(x ; \beta / p), \quad n \geq 0 .
$$

Proof. Apply $\mathcal{D}_{p}$ to both sides of the generating function in (3.1) to get

$$
\sum_{n=0}^{\infty} \mathcal{D}_{p} Q_{n+1}(x ; \beta) t^{n+1}=\frac{2}{1-p} \sum_{n=0}^{\infty} Q_{n}(x ; \beta / p) p^{n / 2} t^{n+1} .
$$

Equating the coefficients of $t^{n+1}$ now leads to (9.1).

The next step is to find an appropriate raising operator.

Lemma 9.2. A raising operator for $Q_{n}(x ; \beta)$ is given by

$$
\frac{1}{\tilde{v}(x ; \beta p)} \mathcal{D}_{p}\left(\tilde{v}(x ; \beta) Q_{n-1}(x ; \beta)\right)=\frac{2\left(p^{n / 2}-p^{-n / 2}\right)}{\beta(1-p)} Q_{n}(x ; \beta p), \quad n \geq 1 .
$$

Proof. A direct computation using the generating function in (3.1) shows that

$$
\begin{aligned}
& \sum_{n=1}^{\infty} \mathcal{D}_{p}\left(\tilde{v}(x ; \beta) Q_{n-1}(x ; \beta)\right) t^{n-1} \\
& \quad=\frac{2}{\beta(1-p)} \tilde{v}(x ; \beta p) \sum_{n=1}^{\infty} Q_{n}(x ; \beta p)\left(p^{n / 2}-p^{-n / 2}\right) t^{n-1},
\end{aligned}
$$

and (9.2) follows by equating the coefficients of $t^{n-1}$.

Combining the lowering and raising operators in Lemma 9.1 and Lemma 9.2 , we get the following result.

Theorem 9.3. The polynomials $Q_{n}(x ; \beta)$ satisfy the $p$-Sturm-Liouville equation

$$
\mathcal{D}_{p}\left(\tilde{v}(x ; \beta / p) \mathcal{D}_{p} Q_{n}(x ; \beta)\right)+\frac{4 \sqrt{p}\left(1-p^{n}\right)}{\beta(1-p)^{2}} \tilde{v}(x ; \beta) Q_{n}(x ; \beta)=0, \quad n \geq 0 .
$$

An alternative proof of the first statement in Theorem [5.1 now goes as follows. Set

$$
h_{n}=-\frac{4 \sqrt{p}}{\beta} \frac{1-p^{n}}{(1-p)^{2}},
$$

and note that this sequence is strictly decreasing in $n$. According to (9.3), we have

$$
\begin{aligned}
\left(h_{n}-\right. & \left.h_{m}\right) \int_{\mathbb{R}} Q_{n}(x ; \beta) Q_{m}(x ; \beta) \tilde{v}(x ; \beta) d x \\
= & \int_{\mathbb{R}} \mathcal{D}_{p}\left(\tilde{v}(x ; \beta / p) \mathcal{D}_{p} Q_{n}(x ; \beta)\right) Q_{m}(x ; \beta) d x \\
& -\int_{\mathbb{R}} \mathcal{D}_{p}\left(\tilde{v}(x ; \beta / p) \mathcal{D}_{p} Q_{m}(x ; \beta)\right) Q_{n}(x ; \beta) d x \\
= & \left\langle\mathcal{D}_{p}\left(\tilde{v}(x ; \beta / p) \mathcal{D}_{p} Q_{n}(x ; \beta)\right), \sqrt{1+x^{2}} Q_{m}(x ; \beta)\right\rangle \\
& -\left\langle\mathcal{D}_{p}\left(\tilde{v}(x ; \beta / p) \mathcal{D}_{p} Q_{m}(x ; \beta)\right), \sqrt{1+x^{2}} Q_{n}(x ; \beta)\right\rangle,
\end{aligned}
$$


and using integration by parts as described in (5.7), this expression reduces to

$$
\begin{aligned}
& -\left\langle\tilde{v}(x ; \beta / p) \mathcal{D}_{p} Q_{n}(x ; \beta), \sqrt{1+x^{2}} \mathcal{D}_{p} Q_{m}(x ; \beta)\right\rangle \\
& +\left\langle\tilde{v}(x ; \beta / p) \mathcal{D}_{p} Q_{m}(x ; \beta), \sqrt{1+x^{2}} \mathcal{D}_{p} Q_{n}(x ; \beta)\right\rangle \\
= & \int_{\mathbb{R}} \mathcal{D}_{p} Q_{n}(x ; \beta) \mathcal{D}_{p} Q_{m}(x ; \beta) \tilde{v}(x ; \beta / p) d x \\
& -\int_{\mathbb{R}} \mathcal{D}_{p} Q_{m}(x ; \beta) \mathcal{D}_{p} Q_{n}(x ; \beta) \tilde{v}(x ; \beta / p) d x=0 .
\end{aligned}
$$

Since $h_{n} \neq h_{m}$ for $n \neq m$, it is proved that $Q_{n}(x ; \beta)$ are orthogonal with respect to $\tilde{v}(x ; \beta)$.

Let $\mathbb{Z}_{a}$ denote the set $\left\{x_{k}(a) \mid k \in \mathbb{Z}\right\}$. Since $\mathbb{Z}_{a p}=\mathbb{Z}_{a}$, we only consider the case $p<a \leq 1$. With respect to the inner product

$$
\langle f, g\rangle_{a}=\sum_{k=-\infty}^{\infty} f\left(x_{k}(a)\right) g\left(x_{k}(a)\right) \sqrt{1+x_{k}^{2}(a)}
$$

on $\ell^{2}\left(\mathbb{Z}_{a}, \sqrt{1+x^{2}}\right)$, integration by parts can be carried out by following the rule:

$$
\left\langle\mathcal{D}_{p} f, g\right\rangle_{a}=-\left\langle f, \mathcal{D}_{p} g\right\rangle_{a \sqrt{p}}
$$

Hence, the $p$-Sturm-Liouville equation (9.3) leads to

$$
\begin{aligned}
\left(h_{n}-\right. & \left.h_{m}\right) \sum_{k=-\infty}^{\infty} Q_{n}\left(x_{k}(a) ; \beta\right) Q_{m}\left(x_{k}(a) ; \beta\right) \tilde{v}\left(x_{k}(a) ; \beta\right) \sqrt{1+x_{k}^{2}(a)} \\
= & \left\langle\mathcal{D}_{p}\left(\tilde{v}(x ; \beta / p) \mathcal{D}_{p} Q_{n}(x ; \beta)\right), Q_{m}(x ; \beta)\right\rangle_{a} \\
& -\left\langle\mathcal{D}_{p}\left(\tilde{v}(x ; \beta / p) \mathcal{D}_{p} Q_{m}(x ; \beta)\right), Q_{n}(x ; \beta)\right\rangle_{a} \\
= & -\left\langle\tilde{v}(x ; \beta / p) \mathcal{D}_{p} Q_{n}(x ; \beta), \mathcal{D}_{p} Q_{m}(x ; \beta)\right\rangle_{a \sqrt{p}} \\
& +\left\langle\tilde{v}(x ; \beta / p) \mathcal{D}_{p} Q_{m}(x ; \beta), \mathcal{D}_{p} Q_{n}(x ; \beta)\right\rangle_{a \sqrt{p}}=0
\end{aligned}
$$

and we have proved that $Q_{n}(x ; \beta)$ are orthogonal with respect to $\tilde{\lambda}_{a}^{(\beta)}$. Exactly the same method can be used to prove that the polynomials $h_{n}(x \mid q)$ are orthogonal with respect to the discrete measures in (1.7).

The essence of Theorem 9.3 is the fact that the polynomials $Q_{n}(x ; \beta)$ are eigenfunctions of the operator $\mathcal{T}$ defined to act on functions in $L^{2}(\mathbb{R}, v(x ; \beta))$ by

$$
\mathcal{T} f(x)=-\frac{1}{\tilde{v}(x ; \beta)} \mathcal{D}_{p}\left(\tilde{v}(x ; \beta / p) \mathcal{D}_{p} f(x)\right) .
$$


The corresponding eigenvalues are given in (9.4). The operator $\mathcal{T}$ is clearly positive since

$$
\begin{aligned}
(\mathcal{T} f(x), f(x))_{v} & =-\int_{\mathbb{R}} \mathcal{D}_{p}\left(\tilde{v}(x ; \beta / p) \mathcal{D}_{p} f(x)\right) \overline{f(x)} d x \\
& =-\left\langle\mathcal{D}_{p}\left(\tilde{v}(x ; \beta / p) \mathcal{D}_{p} f(x)\right), \sqrt{1+x^{2}} f(x)\right\rangle \\
& =\left\langle\tilde{v}(x ; \beta / p) \mathcal{D}_{p} f(x), \sqrt{1+x^{2}} \mathcal{D}_{p} f(x)\right\rangle \\
& =\int_{\mathbb{R}}\left|\mathcal{D}_{p} f(x)\right|^{2} \tilde{v}(x ; \beta / p) d x \geq 0 .
\end{aligned}
$$

However, the polynomials $Q_{n}(x ; \beta)$ are not dense in $L^{2}(\mathbb{R}, v(x ; \beta))$ because the absolutely continuous solution with density $v(x ; \beta)$ is not $N$-extremal. Therefore, we do not have an explicit orthonormal basis for the Hilbert space $L^{2}(\mathbb{R}, v(x ; \beta))$.

The average operator $\mathcal{A}_{p}$ is defined to act on functions on $\mathbb{R}$ by

$$
\mathcal{A}_{p} f(x)=\frac{1}{2}\left(\breve{f}\left(p^{1 / 2} e^{y}\right)+\breve{f}\left(p^{-1 / 2} e^{y}\right)\right)
$$

where again $\breve{f}\left(e^{y}\right)=f(x)$. The reason for introducing $\mathcal{A}_{p}$ is to obtain the $p$-Leibniz rule

$$
\mathcal{D}_{p} f g=\mathcal{D}_{p} f \mathcal{A}_{p} g+\mathcal{A}_{p} f \mathcal{D}_{p} g
$$

which follows from the fact that

$$
(a+b)(c-d)+(a-b)(c+d)=2(a c-b d) \quad \text { for } \quad a, b, c, d \in \mathbb{R} .
$$

It is straightforward to see that

$$
\mathcal{D}_{p} \tilde{v}(x ; \beta)=-\frac{4 x}{\beta \sqrt{p}(1-p)} \tilde{v}(x ; p \beta)
$$

and

$$
\mathcal{A}_{p} \tilde{v}(x ; \beta)=\left(\frac{2 x^{2}+1}{p \beta}+1\right) \tilde{v}(x ; p \beta),
$$

so the $p$-Sturm-Liouville equation (9.3) can be written as

$$
\left(2 x^{2}+1+\beta\right) \mathcal{D}_{p}^{2} Q_{n}(x ; \beta)-\frac{4 x \sqrt{p}}{1-p} \mathcal{A}_{p} \mathcal{D}_{p} Q_{n}(x ; \beta)+\frac{4 \sqrt{p}\left(1-p^{n}\right)}{(1-p)^{2}} Q_{n}(x ; \beta)=0 .
$$

Note that the weight function $\tilde{v}(x ; \beta)$ has disappeared completely and, as such, (9.5) is more general than (9.3).

We are now in a position to derive Bethe Ansatz equations satisfied by the zeros of $Q_{n}(x ; \beta)$; cf. [18.

Theorem 9.4. Let $x_{1}=\sinh y_{1}, \ldots, x_{n}=\sinh y_{n}$ denote the $n$ simple zeros of $Q_{n}(x ; \beta)$. If we set $p=e^{2 \eta}$ and $\beta=e^{2 \gamma}$, then the following $n$ equations are satisfied:

$$
\prod_{\substack{i=1 \\ i \neq j}}^{n} \frac{\sinh \left(\frac{y_{j}-y_{i}}{2}+\eta\right) \cosh \left(\frac{y_{j}+y_{i}}{2}+\eta\right)}{\sinh \left(\frac{y_{j}-y_{i}}{2}-\eta\right) \cosh \left(\frac{y_{j}+y_{i}}{2}-\eta\right)}=e^{-2 y_{j}} \frac{\cosh \left(y_{j}+\gamma\right)}{\cosh \left(y_{j}-\gamma\right)}, \quad j=1, \ldots, n .
$$

Proof. Let $c_{n}$ denote the leading coefficient of $Q_{n}(x ; \beta)$ and define

$$
f(x):=\frac{1}{c_{n}} Q_{n}(x ; \beta)=\prod_{i=1}^{n}\left(x-x_{i}\right) .
$$


A tiresome computation shows that

$$
\begin{aligned}
& \left.\mathcal{D}_{p}^{2} f(x)\right|_{x=x_{j}} \\
& =\frac{2}{(\sqrt{p}-1 / \sqrt{p})^{2}}\left(\frac{\prod_{i}\left(\frac{e^{y_{j}} p-e^{-y_{j}} / p}{2}-x_{i}\right)}{e^{y_{j}} \sqrt{p}-e^{-y_{j}} / \sqrt{p}}+\frac{\prod_{i}\left(\frac{e^{y_{j}} / p-e^{-y_{j}} p}{2}-x_{i}\right)}{e^{y_{j}} / \sqrt{p}-e^{-y_{j}} \sqrt{p}}\right) \frac{2}{e^{y_{j}}+e^{-y_{j}}}
\end{aligned}
$$

and similarly

$$
\begin{aligned}
& \left.\mathcal{A}_{p} \mathcal{D}_{p} f(x)\right|_{x=x_{j}} \\
& \quad=\frac{1}{\sqrt{p}-1 / \sqrt{p}}\left(\frac{\prod_{i}\left(\frac{e^{y_{j}} p-e^{-y_{j}} / p}{2}-x_{i}\right)}{e^{y_{j}} \sqrt{p}-e^{-y_{j}} / \sqrt{p}}-\frac{\prod_{i}\left(\frac{e^{y_{j}} / p-e^{-y_{j}} p}{2}-x_{i}\right)}{e^{y_{j}} / \sqrt{p}-e^{-y_{j}} \sqrt{p}}\right) .
\end{aligned}
$$

According to (9.5), we thus have

$$
\begin{aligned}
& \left(2 x_{j}^{2}+1+\beta\right) \frac{2}{(\sqrt{p}-1 / \sqrt{p})^{2}} \\
& \quad \times\left(\frac{\prod_{i}\left(\frac{e^{y_{j}} p-e^{-y_{j}} / p}{2}-x_{i}\right)}{e^{y_{j}} \sqrt{p}-e^{-y_{j}} / \sqrt{p}}+\frac{\prod_{i}\left(\frac{e^{y_{j}} / p-e^{-y_{j}} p}{2}-x_{i}\right)}{e^{y_{j}} / \sqrt{p}-e^{-y_{j}} \sqrt{p}}\right) \frac{2}{e^{y_{j}}+e^{-y_{j}}} \\
& =\frac{4 x_{j} \sqrt{p}}{1-p} \frac{1}{\sqrt{p}-1 / \sqrt{p}}\left(\frac{\prod_{i}\left(\frac{e^{y_{j}} p-e^{-y_{j}} / p}{2}-x_{i}\right)}{e^{y_{j}} \sqrt{p}-e^{-y_{j}} / \sqrt{p}}-\frac{\prod_{i}\left(\frac{e^{y_{j}} / p-e^{-y_{j}} p}{2}-x_{i}\right)}{e^{y_{j}} / \sqrt{p}-e^{-y_{j}} \sqrt{p}}\right)
\end{aligned}
$$

or

$$
\begin{gathered}
\left(\frac{e^{2 y_{j}}+e^{-2 y_{j}}}{2}+\beta\right)\left(\frac{\prod_{i}\left(\frac{e^{y_{j}} p-e^{-y_{j}} / p}{2}-x_{i}\right)}{e^{y_{j}} \sqrt{p}-e^{-y_{j}} / \sqrt{p}}+\frac{\prod_{i}\left(\frac{e^{y_{j}} / p-e^{-y_{j}} p}{2}-x_{i}\right)}{e^{y_{j}} / \sqrt{p}-e^{-y_{j}} \sqrt{p}}\right) \\
=\frac{e^{2 y_{j}}-e^{-2 y_{j}}}{2}\left(\frac{\prod_{i}\left(\frac{e^{y_{j}} p-e^{-y_{j}} / p}{2}-x_{i}\right)}{e^{y_{j}} \sqrt{p}-e^{-y_{j}} / \sqrt{p}}-\frac{\prod_{i}\left(\frac{e^{y_{j}} / p-e^{-y_{j}} p}{2}-x_{i}\right)}{e^{y_{j}} / \sqrt{p}-e^{-y_{j}} \sqrt{p}}\right) .
\end{gathered}
$$

Hence,

$$
\begin{aligned}
\left(e^{2 y_{j}}\right. & +\beta)\left(e^{y_{j}} / \sqrt{p}+e^{-y_{j}} \sqrt{p}\right) \prod_{i=1}^{n}\left(\frac{e^{y_{j}} p-e^{-y_{j}} / p}{2}-x_{i}\right) \\
& =-\left(e^{-2 y_{j}}+\beta\right)\left(e^{y_{j}} \sqrt{p}+e^{-y_{j}} / \sqrt{p}\right) \prod_{i=1}^{n}\left(\frac{e^{y_{j}} / p-e^{-y_{j}} p}{2}-x_{i}\right),
\end{aligned}
$$

that is,

$$
\prod_{i=1}^{n} \frac{\left(e^{y_{j}} p-e^{-y_{j}} / p\right) / 2-x_{i}}{\left(e^{y_{j}} / p-e^{-y_{j}} p\right) / 2-x_{i}}=-\frac{\left(e^{-2 y_{j}}+\beta\right)\left(e^{y_{j}} \sqrt{p}+e^{-y_{j}} / \sqrt{p}\right)}{\left(e^{2 y_{j}}+\beta\right)\left(e^{y_{j}} / \sqrt{p}+e^{-y_{j}} \sqrt{p}\right)} .
$$

With $p=e^{2 \eta}$ and $\beta=e^{2 \gamma}$, the above expression can be written as

$$
\prod_{i=1}^{n} \frac{\sinh \left(\frac{y_{j}-y_{i}}{2}+\eta\right) \cosh \left(\frac{y_{j}+y_{i}}{2}+\eta\right)}{\sinh \left(\frac{y_{j}-y_{i}}{2}-\eta\right) \cosh \left(\frac{y_{j}+y_{i}}{2}-\eta\right)}=-e^{-2 y_{j}} \frac{\cosh \left(y_{j}+\gamma\right) \cosh \left(y_{j}+\eta\right)}{\cosh \left(y_{j}-\gamma\right) \cosh \left(y_{j}-\eta\right)},
$$

and the theorem is proved once we remove the factor corresponding to $i=j$.

For more information about the connection between $q$-Sturm-Liouville problems and Bethe Ansatz equations, the reader is referred to [12. 


\section{A right inverse to the divided difference operator $\mathcal{D}_{p}$}

In Section 5 and Section 9 we have seen that the divided difference operator $\mathcal{D}_{q}$ (or $\mathcal{D}_{p}$ ) is a lowering operator for the polynomials $h_{n}(x \mid q)$ and $Q_{n}(x ; \beta)$. In this section we establish a right inverse $\mathcal{D}_{q}^{-1}\left(\right.$ or $\mathcal{D}_{p}^{-1}$ ) on appropriate $L^{2}$-spaces.

For an indeterminate moment problem on the real line it is well known that the polynomials are dense in $L^{2}(\mathbb{R}, \mu)$ if and only if the measure $\mu$ is $N$-extremal. So in the case of the $q^{-1}$-Hermite moment problem, the polynomials

$$
P_{n}(x)=\frac{q^{n(n+1) / 4}}{\sqrt{(q ; q)_{n}}} h_{n}(x \mid q)
$$

form an orthonormal basis for $L^{2}\left(\mathbb{R}, \nu_{a}\right)$ exactly when $\nu_{a}$ has the form (1.7) (which we shall assume). Any function $f \in L^{2}\left(\mathbb{R}, \nu_{a}\right)$ can thus be written as

$$
f(x) \sim \sum_{n=0}^{\infty} f_{n} P_{n}(x)
$$

for some sequence $\left(f_{n}\right) \in \ell^{2}$ and since

$$
\mathcal{D}_{q} P_{n}(x)=-\frac{2 \sqrt{1-q^{n}}}{\sqrt{q}-1 / \sqrt{q}} P_{n-1}(x),
$$

we see that

$$
\mathcal{D}_{q} f(x) \sim \frac{-2}{\sqrt{q}-1 / \sqrt{q}} \sum_{n=0}^{\infty} f_{n+1} \sqrt{1-q^{n+1}} P_{n}(x) .
$$

In other words, we can think of $\mathcal{D}_{q}$ as a bounded operator on $L^{2}\left(\mathbb{R}, \nu_{a}\right)$. It is readily seen that $\mathcal{D}_{q}$ is onto, for if $g \in L^{2}\left(\mathbb{R}, \nu_{a}\right)$ has the form

$$
g(x) \sim \sum_{n=0}^{\infty} g_{n} P_{n}(x)
$$

then $\mathcal{D}_{q} f(x)=g(x)$ with $f$ as in (10.1) and

$$
f_{n}=-\frac{\sqrt{q}-1 / \sqrt{q}}{2} \frac{g_{n-1}}{\sqrt{1-q^{n}}}
$$

But $\mathcal{D}_{q}$ is clearly not one-to-one. So we can only have hope of finding a right inverse to $\mathcal{D}_{q}$, that is, an operator $\mathcal{D}_{q}^{-1}$ on $L^{2}\left(\mathbb{R}, \nu_{a}\right)$ so that

$$
\mathcal{D}_{q} \mathcal{D}_{q}^{-1}=I \text {. }
$$

It is straightforward how to define $\mathcal{D}_{q}^{-1}$. With $g$ as in (10.2), the operator $\mathcal{D}_{q}^{-1}$ is given by

$$
\begin{aligned}
\mathcal{D}_{q}^{-1} g(x) & \sim-\frac{\sqrt{q}-1 / \sqrt{q}}{2} \sum_{n=1}^{\infty} \frac{g_{n-1}}{\sqrt{1-q^{n}}} P_{n}(x) \\
& =-\frac{\sqrt{q}-1 / \sqrt{q}}{2} \sum_{n=1}^{\infty} \frac{\int_{\mathbb{R}} g\left(x^{\prime}\right) P_{n-1}\left(x^{\prime}\right) d \nu_{a}\left(x^{\prime}\right)}{\sqrt{1-q^{n}}} P_{n}(x) \\
& =-\frac{\sqrt{q}-1 / \sqrt{q}}{2} \int_{\mathbb{R}} g\left(x^{\prime}\right) \sum_{n=1}^{\infty} \frac{P_{n-1}\left(x^{\prime}\right) P_{n}(x)}{\sqrt{1-q^{n}}} d \nu_{a}\left(x^{\prime}\right),
\end{aligned}
$$


and this means that $\mathcal{D}_{q}^{-1}$ is an integral operator with kernel

$$
K\left(x, x^{\prime}\right)=\sum_{n=1}^{\infty} \frac{P_{n}(x) P_{n-1}\left(x^{\prime}\right)}{\sqrt{1-q^{n}}}=\sum_{n=1}^{\infty} \frac{q^{n^{2} / 2}}{(q ; q)_{n}} h_{n}(x \mid q) h_{n-1}\left(x^{\prime} \mid q\right) .
$$

Since $\left(P_{n}(x)\right) \in \ell^{2}$ for all $x \in \mathbb{C}$, the kernel in (10.3) is convergent in $L^{2}\left(\mathbb{R}, \nu_{a}\right)$ as a function of $x^{\prime}$. The change of summation and integration can therefore easily be justified.

Theorem 10.1. The kernel in (10.3) is explicitly given by

$$
\begin{aligned}
\sum_{n=1}^{\infty} & \frac{q^{n^{2} / 2}}{(q ; q)_{n}} h_{n}(x \mid q) h_{n-1}\left(x^{\prime} \mid q\right) \\
= & \frac{2\left(-\sqrt{q} e^{y+y^{\prime}},-\sqrt{q} e^{-y-y^{\prime}}, \sqrt{q} e^{y-y^{\prime}}, \sqrt{q} e^{-y+y^{\prime}} ; q\right)_{\infty}}{(q ; q)_{\infty}} \\
& \times \sum_{n=0}^{\infty} \frac{(q ; q)_{2 n}\left(\sinh y-q^{n+1 / 2} \sinh y^{\prime}\right) q^{n+1 / 2}}{\left(-\sqrt{q} e^{y+y^{\prime}},-\sqrt{q} e^{-y-y^{\prime}}, \sqrt{q} e^{y-y^{\prime}}, \sqrt{q} e^{-y+y^{\prime}} ; q\right)_{n+1}} .
\end{aligned}
$$

Proof. The idea of the proof is to apply $\mathcal{D}_{q}$ with respect to $x^{\prime}$ to the Poisson kernel. Applying $\mathcal{D}_{q}$ (wrt. $x^{\prime}$ ) to the left-hand side in (1.5) leads to

$$
\frac{-2}{\sqrt{q}-1 / \sqrt{q}} \sum_{n=1}^{\infty} \frac{q^{n^{2} / 2-n}}{(q ; q)_{n-1}} h_{n}(x \mid q) h_{n-1}\left(x^{\prime} \mid q\right) t^{n}
$$

or

$$
\frac{-2}{\sqrt{q}-1 / \sqrt{q}}\left\{H\left(x, x^{\prime} ; t / q\right)-H\left(x, x^{\prime} ; t\right)\right\}
$$

if we set

$$
H\left(x, x^{\prime} ; t\right)=\sum_{n=1}^{\infty} \frac{q^{n^{2} / 2}}{(q ; q)_{n}} h_{n}(x \mid q) h_{n-1}\left(x^{\prime} \mid q\right) t^{n} .
$$

Applying $\mathcal{D}_{q}$ (wrt. $\left.x^{\prime}\right)$ to the right-hand side gives, after some computations,

$$
\begin{aligned}
& \frac{-2}{\sqrt{q}-1 / \sqrt{q}} \frac{2 t}{\sqrt{q}}\left(\sinh y-\frac{t}{\sqrt{q}} \sinh y^{\prime}\right) \\
& \times \frac{\left(-t \sqrt{q} e^{y+y^{\prime}},-t \sqrt{q} e^{-y-y^{\prime}}, t \sqrt{q} e^{y-y^{\prime}}, t \sqrt{q} e^{-y+y^{\prime}} ; q\right)_{\infty}}{\left(t^{2} / q ; q\right)_{\infty}} .
\end{aligned}
$$

In other words,

$$
\begin{aligned}
& H\left(x, x^{\prime} ; t / q\right)-H\left(x, x^{\prime} ; t\right) \\
& =\frac{2 t}{\sqrt{q}}\left(\sinh y-\frac{t}{\sqrt{q}} \sinh y^{\prime}\right) \frac{\left(-t \sqrt{q} e^{y+y^{\prime}},-t \sqrt{q} e^{-y-y^{\prime}}, t \sqrt{q} e^{y-y^{\prime}}, t \sqrt{q} e^{-y+y^{\prime}} ; q\right)_{\infty}}{\left(t^{2} / q ; q\right)_{\infty}},
\end{aligned}
$$


and since $H\left(x, x^{\prime} ; 0\right)=0$, we have

$$
\begin{aligned}
H\left(x, x^{\prime} ; t\right)= & \sum_{n=1}^{\infty}\left\{H\left(x, x^{\prime} ; t q^{n-1}\right)-H\left(x, x^{\prime} ; t q^{n}\right)\right\} \\
= & 2 \sum_{n=1}^{\infty}\left(\sinh y-t q^{n-1 / 2} \sinh y^{\prime}\right) t q^{n-1 / 2} \\
& \times \frac{\left(-t q^{n+1 / 2} e^{y+y^{\prime}},-t q^{n+1 / 2} e^{-y-y^{\prime}}, t q^{n+1 / 2} e^{y-y^{\prime}}, t q^{n+1 / 2} e^{-y+y^{\prime}} ; q\right)_{\infty}}{\left(t^{2} q^{2 n-1} ; q\right)_{\infty}} \\
= & \frac{2\left(-t \sqrt{q} e^{y+y^{\prime}},-t \sqrt{q} e^{-y-y^{\prime}}, t \sqrt{q} e^{y-y^{\prime}}, t \sqrt{q} e^{-y+y^{\prime}} ; q\right)_{\infty}}{\left(t^{2} q ; q\right)_{\infty}} \\
& \times \sum_{n=1}^{\infty} \frac{\left(t^{2} q ; q\right)_{2 n-2}\left(\sinh y-t q^{n-1 / 2} \sinh y^{\prime}\right) t q^{n-1 / 2}}{\left(-t \sqrt{q} e^{y+y^{\prime}},-t \sqrt{q} e^{-y-y^{\prime}}, t \sqrt{q} e^{y-y^{\prime}}, t \sqrt{q} e^{-y+y^{\prime}} ; q\right)_{n}} .
\end{aligned}
$$

It is only left to set $t=1$ and shift the summation.

For the moment problem associated with the polynomials $Q_{n}(x ; \beta)$, the situation is almost the same. However, since

$$
\mathcal{D}_{p} P_{n}(x ; \beta)=\frac{2 \sqrt{p}}{1-p} \sqrt{\frac{1-p^{n}}{\beta+1}} P_{n-1}(x ; \beta / p),
$$

the operator $\mathcal{D}_{p}$ maps $L^{2}\left(\mathbb{R}, \nu_{a}^{(\beta)}\right)$ into $L^{2}\left(\mathbb{R}, \nu_{a}^{(\beta / p)}\right)$, and the right inverse $\mathcal{D}_{p}^{-1}$ is defined on $L^{2}\left(\mathbb{R}, \nu_{a}^{(\beta / p)}\right)$ as the integral operator with kernel

$$
K\left(x, x^{\prime} ; \beta\right)=\sum_{n=1}^{\infty} \frac{P_{n}(x ; \beta) P_{n-1}\left(x^{\prime} ; \beta / p\right)}{\sqrt{1-p^{n}}} .
$$

Theorem 10.2. The kernel in (10.4) is explicitly given by

$$
\begin{aligned}
\sum_{n=1}^{\infty} & \frac{P_{n}(x ; \beta) P_{n-1}\left(x^{\prime} ; \beta / p\right)}{\sqrt{1-p^{n}}} \\
= & -\frac{i \sqrt{p}}{2} \frac{\left(-i e^{y} / \sqrt{\beta}, i e^{-y} / \sqrt{\beta}, i \sqrt{p} e^{y^{\prime}} / \sqrt{\beta},-i \sqrt{p} e^{-y^{\prime}} / \sqrt{\beta}\right)_{\infty}}{(-p / \beta ; p)_{\infty} \sqrt{1+1 / \beta}} \frac{\left(p ; p^{2}\right)_{\infty}}{\left(p^{2} ; p^{2}\right)_{\infty}} \\
& \times \sum_{n=0}^{\infty} \frac{\left(p^{2} ; p^{2}\right)_{n}}{\left(i \sqrt{p} e^{y^{\prime}} / \sqrt{\beta},-i \sqrt{p} e^{-y^{\prime}} / \sqrt{\beta}\right)_{n}} p^{n} \sum_{k=0}^{n} \frac{\left(i e^{y} / \sqrt{\beta},-i e^{-y} / \sqrt{\beta} ; p\right)_{k}}{\left(p^{2} ; p^{2}\right)_{k}} p^{k} \\
& + \text { a similar term with y replaced by }-y \text { and } y^{\prime} \text { replaced by }-y^{\prime} .
\end{aligned}
$$

Proof. The same procedure as in the proof of Theorem 10.1 can be carried out. The details are left to the reader.

If we compare $\mathcal{D}_{q}$ (or $\mathcal{D}_{p}$ ) with differentiation $d / d x$, it is remarkable that $\mathcal{D}_{q}$ is bounded on $L^{2}\left(\mathbb{R}, \nu_{a}\right)$ whereas $d / d x$ is unbounded. Also, the Askey-Wilson operator is known to be unbounded on the $L^{2}$-space weighted by the weight function for the Askey-Wilson polynomials.

Since $\mathcal{D}_{q}$ is a $q$-analogue of differentiation, we can think of $\mathcal{D}_{q}^{-1}$ as a $q$-analogue of integration. Thus, for $f \in L^{2}\left(\mathbb{R}, \nu_{a}\right)$ we have the following $q$-analogue of the 
definite integral:

$$
\int_{a}^{b} f(x) \breve{d}_{q} x=-\frac{\sqrt{q}-1 / \sqrt{q}}{2} \int_{\mathbb{R}} f(x)(K(b, x)-K(a, x)) d \nu_{a}(x)
$$

where the kernel $K$ is computed in Theorem 10.1.

\section{REFERENCES}

[1] N. I. Akhiezer, "The classical moment problem," Oliver and Boyd, Edinburgh and London, 1965.

[2] R. Askey, Continuous $q$-Hermite polynomials when $q>1$, in: " $q$-Series and Partitions," D. Stanton, (ed.), IMA Vol. Math. Appl., Springer-Verlag, 1989, 151-158. MR1019849 (90h:33019)

[3] G. E. Andrews, R. Askey, and R. Roy, "Special Functions," Cambridge University Press, Cambridge, 1999. MR.1688958 (2000g:33001)

[4] R. Askey and M. E. H. Ismail, Recurrence relations, continued fractions and orthogonal polynomials, Mem. Amer. Math. Soc. 300 (1984). MR0743545 (85g:33008)

[5] R. Askey and R. Roy, More q-beta integrals, Rocky Mountain J. Math. 16 (1986), 365-372. MR0843057 (87f:33002)

[6] C. Berg and J. P. R. Christensen, Density questions in the classical theory of moments, Ann. Inst. Fourier 31 (1981), 99-114. MR0638619 (84i:44006)

[7] C. Berg and M. E. H. Ismail, $q$-Hermite polynomials and classical orthogonal polynomials, Can. J. Math Vol. 48 (1996), 43-63. MR1382475 (97b:33020)

[8] B. M. Brown and M. E. H. Ismail, A right inverse of the Askey-Wilson operator, Proc. Amer. Math. Soc. Vol. 123 (1995), 2071-2079. MR1273478 (95i:33019)

[9] T. S. Chihara and M. E. H. Ismail, Extremal measures for a system of orthogonal polynomials, Constr. Approx. 9 (1993), 111-119. MR1198526 (94b:33020)

[10] G. Gasper and M. Rahman, Basic hypergeometric series, Cambridge University Press, Cambridge, 1990. MR1052153(91d:33034)

[11] M. E. H. Ismail, Ladder operators for $q^{-1}$-Hermite polynomials, C. R. Math. Rep. Acad. Sci. Canada 15 (1993), 261-266. MR1260071 (95b:33050)

[12] M. E. H. Ismail, S. Lin and S. Roan, Bethe Ansatz and $q$-Sturm-Liouville problems, J. Math. Phys., to appear.

[13] M. E. H. Ismail and D. R. Masson, $q$-Hermite polynomials, biorthogonal rational functions and $q$-beta integrals, Trans. Amer. Math. Soc 346 (1994), 63-116. MR1264148 (96a:33022)

[14] M. E. H. Ismail and M. Rahman, An inverse to the Askey-Wilson operator, Rocky Mountain J. Math. 32 (2002), 657-678. MR1934910 (2003m:33022)

[15] M. E. H. Ismail and D. Stanton, Classical orthogonal polynomials as moments, Can. J. Math. 49 (1997), 520-542. MR 1451259 (98f:33033)

[16] M. E. H. Ismail and D. Stanton, $q$-integral and moment representations for $q$-orthogonal polynomials, Can. J. Math. 54 (2002), 709-735. MR1913916(2003k:33024)

[17] R. Koekoek and R. F. Swarttouw, "The Askey-scheme of hypergeometric orthogonal polynomials and its $q$-analogue," Report no. 98-17, TU-Delft, 1998.

[18] V. E. Korepin, N. M. Bogoliubov, and A. G. Izergin, "Quantum Inverse Scattering Method and Correlation Functions," Cambridge Univesity Press, Cambridge, 1993. MR 1245942 (95b:81224)

[19] J. Shohat and J. D. Tamarkin, The Problem of Moments, revised edition, American Mathematical Society, Providence, 1950. MR0008438 (5:5c)

[20] B. Simon, The classical moment as a selfadjoint finite difference operator, Adv. Math. 137 (1998), 82-203. MR.1627806 (2001e:47020)

[21] M. H. Stone, Linear Transformations in Hilbert Space and Their Application to Analysis, American Mathematical Society, Providence, 1932. MR1451877 (99k:47001)

Department of Mathematics, University of Copenhagen, Universitetsparken 5, 2100 København, Denmark

E-mail address: stordal@math.ku.dk

Department of Mathematics, University of Central Florida, Orlando, Florida 32816 E-mail address: ismail@math.ucf.edu 